Unpicking the interplay of turbulence, diffusion and thermophysics in cryogenic jets at supercritical pressures

\title{
Unpicking the interplay of turbulence, diffusion and thermophysics in cryogenic jets at supercritical pressures
}

Jaya Vignesh Madana Gopal, ${ }^{1}$ Giovanni Tretola, ${ }^{1}$ Robert Morgan, ${ }^{1}$ Guillaume de Sercey, ${ }^{1}$ Grazia Lamanna, ${ }^{2}$ and Konstantina Vogiatzaki ${ }^{1 a)}$

${ }^{1)}$ Advanced Engineering Center, University of Brighton, Lewis Road, BN2 4AT, Brighton,

United Kingdom

${ }^{2)}$ Institute of Aerospace Thermodynamics, University of Stuttgart, Pfaffenwaldring 31, 70569 Stuttgart,

Germany

(Dated: 17 June 2021)

Cryogenic supercritical fluids represent an intriguing category of fluids that combine mechanical and thermophysical properties of both ultra low temperature conditions as well as phenomena taking place well above the critical point. Recent research has demonstrated that it is a common misconception to consider the supercritical state as one homogeneous state. Instead these fluids consist of two to four liquid and gas like phases, each with their own unique characteristics. In our work we investigate numerically single specie cryogenic fluid jets -initially at subcritical temperatureswhich are injected into a supercritical environment (both the pressure and temperature exceed the thermodynamic critical state). For the investigation, a new solver namely "CoolFoam" has been developed which is designed for compressible non-isothermal two-fluid simulations where diffusive transport of heat and/or mass are accounted for. Real fluid thermodynamics are modelled using a polynomial fitting approach developed in our previous work. We introduce also a new phase characterisation framework based on the association of phases with specific temperature ranges (rather than using a single line like the Widom line) which allowed us to better identify similarity effects between the various conditions. We analyse the inter-dependence of the underlying phenomena: density gradient and diffusive mass transport (molecular and thermo(Soret)-diffusion) and turbulence. We also compared supercritical $\mathrm{N}_{2}$ with subcritical liquid and gas jets to highlight potential differences with respect to how these jets behave. We find that the jet dynamics are largely dictated by the thermodynamic transition of the injected fluid and the associated variation in thermophysical properties.

\section{INTRODUCTION}

Cryogenic fluids represent an intriguing category of fluids. Although gases at atmospheric conditions, they find applications mostly in liquid state that they acquire at low temperatures and high pressures. In the last decade, interest for the use of these fluids in various engineering and scientific applications has increased exponentially. Apart from traditional applications (cryogenic $\mathrm{O}_{2}$ and $\mathrm{H}_{2}$ are already widely used in cryogenic rocket engines), new emerging technologies rely on ultra low temperature conditions. Such applications include a new high efficiency internal combustion (IC) engine (the so called "Cryopower" split cycle engine ${ }^{1}$ that breaks the limit of $60 \%$ efficiency due to isothermal compression succeeded by liquid nitrogen $\left(\mathrm{LN}_{2}\right)$ injection into the compression cylinder, as well as the new generation of cryogenic rocket engines that use liquid $\mathrm{CH}_{4}$ (such as SpaceX Raptor ${ }^{2}$ and Blue Origin BE-4 $\left.{ }^{3}\right)$. Another interesting application is cryotherapy ${ }^{4,5}$ where the extreme cold produced by $\mathrm{LN}_{2}$ vaporisation is used to destroy abnormal tissues. $\mathrm{LN}_{2}$ is applied directly to the cancer cells with a spraying device.

In most applications, cryogenic jets are injected in high pressures and temperatures. While these conditions are beyond the critical point of the cryogenic fluids, in practice, the injected fluids are stored and introduced at very low temperature in a subcritical cryogenic liquid state. Thus, the transition

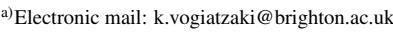

to the supercritical state does not occur immediately. Instead the jets undergo a gradual phase change process with various intermediate states before becoming fully supercritical. The process is further complicated by the change in surface tension observed during the transition. When the cryogenic fluid is in liquid state, surface tension is one of the dominant mechanisms that controls the development of the initial instabilities in the same way as with any other liquids. In our previous work (Ref. 6) we showed that subcritical cryogenic jets obey the existing breakup regime classification by Liu and Altan for liquid jets based on Reynold (Re), Weber (We) and Ohnesorge $(\mathrm{Oh})$ numbers. In common fluids these instabilities are also linked to turbulence generation given high enough injection Re numbers. As the cryogenic fluid transitions to a supercritical state, surface tension reduces and ceases to exist once the fluid reaches critical temperature.

The complex cryogenic jet fluid dynamics were well portrayed more than twenty years ago by the experiments of Mayer et al. ${ }^{8-10}$, Chehroudi et al. ${ }^{11}$ and Oschwald et al. ${ }^{12,13}$. These experiments provided clear evidence of droplets and ligaments in subcritical environment, but exhibited gas like diffusion while possessing liquid like density at supercritical pressures. With increase in the supercritical pressure, the diffusive nature was further amplified. This behaviour is very similar to any other fluid transitioning beyond the critical point as demonstrated already since 1971 from the experimental work on supercritical $\mathrm{CO}_{2}$ by Newman and Brzustowski ${ }^{14}$. Recently new evidence relevant to the behaviour of cryogenic jets has been produced based both on numerical and experimental efforts. Banuti has presented a detailed thermo- 
dynamic analysis of supercritical fluids and jets ${ }^{15,16}$ as well as molecular dynamic computations presenting the molecular distribution of a fluid at subcritical, transcritical and supercritical states ${ }^{17}$. Moreover, there has been a surge in experiments on cryogenic flashing by Lamanna et al. ${ }^{18-22}$. Regardless of these new literature, fundamental questions around the complex interplay of turbulence, diffusion and thermophysical properties in cryogenic jets at supercritical conditions still remain open.

In this paper, we provide new insight into the turbulence development and diffusive transport characteristics of supercritical cryogenic jets. We will focus our analysis to single specie cryogenic fluids, as phase transition in mixtures is governed by more complex mechanisms $\mathrm{s}^{23,24}$ and the research is still in preliminary stages with respect to the thermophysical properties of mixtures of supercritical fluids ${ }^{17,25}$. Our aim is to provide answers to three fundamental questions that are still open regarding the nature of these jets:

1. What is the dominant mechanism in the jet development (fluid mechanics or thermodynamics)?

2. Since these jets are commonly characterised by high $\mathrm{Re}$ numbers, do they manifest turbulent characteristics similar to turbulent jets at atmospheric pressures?

3. Given that the supercrticial state in reality consists of liquid like and gas like states do the jets behave more like liquid or gas jets?

In order to answer these questions, we simulate a series of cryogenic $\mathrm{LN}_{2}$ jet injections into a chamber filled with gaseous $\mathrm{N}_{2}$, using our in-house developed solver 'CoolFoam' which is able to account for the diffusive transport of mass and energy as well as phase changes at supercritical conditions. First, the classification of phases and supercritical pseudo-phases in a cryogenic jet is introduced, based on their isobaric thermophysical property variation against temperature. Then the complete numerical simulation setup including the solver's governing equations is presented. The simulation cases consist of chamber pressures in the range of 1.8 to 6 times the critical pressure $\left(P_{c r}\right)$ of nitrogen and two injection velocities $(2$ and $10 \mathrm{~m} / \mathrm{s})$. These conditions correspond to a Re numbers range of 58000 to 290000 which allows us to investigate the role of turbulence at various levels and its effect on the dynamics of a transitioning to high supercritical cryogenic jet. In the Results section we focus on three aspect of these jets a) We discuss the instantaneous and mean profile behaviour and phenomena of self similarity are identified for the first time b) We analyse the jet fluctuations in an effort to shed light to the mechanisms causing them (turbulence or thermophysically driven) c) We investigate the effect that the density gradients have on the turbulence both close to the injection point as well as further downstream. For the first time we provide details on the physical link between these gradients and the rise of fluctuations.

\section{CLASSIFICATION OF PHASES AND SUPERCRITICAL PSEUDO-PHASES IN A CRYOGENIC JET}

At subcritical chamber pressures -as with any other fluidthe cryogenic jet enters the chamber at liquid state and vaporises due to evaporation and/or boiling, depending on the chamber conditions. For pure fluids at these conditions, a clear line exists in the state diagram that separates the states where the cryogenic fluid exists as liquid and when it transitions to gas. Though, such a clear singular separation between the states ends at the critical point. After the critical point the liquid transitions to supercritical state, which although is misleadingly considered in some earlier literature as one homogeneous state, theoretical, and experimental evidence ${ }^{25-28}$ show that it can be in reality divided into regions with liquid-like and gas-like properties, by an extension of the saturation line. An initial simple separation of these regions was provided by Banuti ${ }^{15}$ who classified the supercritical phases based on the pseudoboiling temperature: Liquid-like $\left(T<T_{p b}\right)$, transitional $\left(T \approx T_{p b}\right)$ and gas-like $\left(T>T_{p b}\right)$. Recently, more insight into these new regions has been provided both at macroscopic (for example from the analysis of thermodynamic data available at $\mathrm{NIST}^{29}$ ) as well as at microscopic level based on molecular dynamic simulations ${ }^{17,25}$. In these studies rigorous approaches of extracting cross over lines have been suggested reflecting either a thermodynamic cross over or a dynamic/ microscopic cross-over. There are various cross-over lines that have been suggested in the literature including the well known Widom line ${ }^{17,30}$ as well as other less commonly used lines (Fisher-Widom line ${ }^{31}$, Nishikawa's ridge ${ }^{32}$ and Frenkel line $^{33}$ )

Here we suggest an alternative way of categorisation of the phases and supercritical pseudo-phases based on identifying temperature ranges over which the changes of the properties occur instead of only focusing on the maxima of the thermodynamic response functions as with previously suggested lines(see 34 for thermodynamic response functions). The general categorisation of all phases and supercritical pseudophases at both subcritical and supercritical pressures is explained in Table I and the categorisation at supercritical pressures alone is demonstrated in Fig. 2 using $\mathrm{N}_{2}$. Based on this isobaric thermophysical properties' variation against temperature, we classify a cryogenic fluid into: liquid, transitional, dense gas-like, ideal gas-like and gas. This classification will be used throughout this paper.

- The liquid phase exists up to the critical temperature $\left(T_{c r}\right)$ and an important characteristic of this phase is the existence of surface tension.

- The transitional phase which corresponds to the drastic changes in all thermophysical properties, encapsulates the pseudoboiling temperature $\left(T_{p b}\right)$. The transitional phase starts from critical temperature and ends at the temperature where the heat capacity falls back to the value at the critical temperature. This region which consists of considerable variation in thermophysical properties expands to a wider range of temperatures at higher supercritical pressures. 


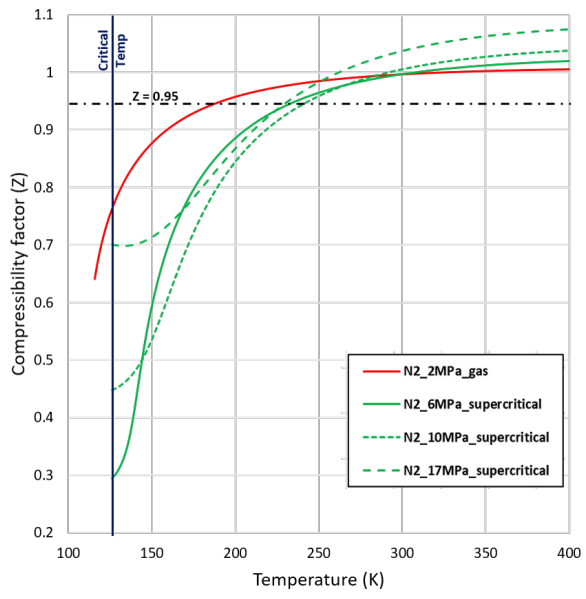

FIG. 1. Compressibility factor $(\mathrm{Z})$ of nitrogen $\left(\mathrm{N}_{2}\right)$ gas against temperature at isobaric pressure of $2 \mathrm{MPa}\left(0.6 \mathrm{P}_{c r}\right)$ and $\mathrm{N}_{2}$ supercritical fluid at $6 \mathrm{MPa}\left(1.8 P_{c r}\right), 10 \mathrm{MPa}\left(3 P_{c r}\right)$ and $17 \mathrm{MPa}\left(5 P_{c r}\right)$ calculated using NIST data of thermophysical properties.

- When the compressibility factor attains a value of 0.95 then the gas-like (supercritical) phase behaves more like an ideal gas. It can be observed in Fig. 1 for all the pressures $(6 \mathrm{MPa}-17 \mathrm{MPa})$ the compressibility factor of $\mathrm{N}_{2}$ possesses values above 0.95 beyond $250 \mathrm{~K}$. Thus, we consider the (ideal) gas-like phase to exist above $250 \mathrm{~K}$ for all supercritical pressures.

- The dense gas-like phase is classified as the phase which exists between the end of transitional phase and start of (ideal) gas-like phase. Though the thermophysical properties of the dense gas-like phase is much close to the (ideal) gas-like phase, the variation in thermophysical properties with temperature do not follow the usual trend of gaseous phase and hence the existing models for thermophysical properties of gases can not be utilised for this dense gas-like phase.

- Gas phase is absent at supercritical pressures and is present only at subcritical pressures.

The suggested categorisation has three main advantages in comparison to the use of a single line to seperate the phase and supercritical pseudo-phases: First, it reflects better the dynamics of the transition from a liquid like to gas like region that does not happen immediately by the crossing of the line but instead occurs over a range of temperatures. At the same time it allows to acquire a better understanding of a range of property variation that occurs during this transition. Secondly, this approach is simpler avoiding ambiguities and complexities associated with the exact derivations of cross-over lines. Thirdly, the use of this separation in the context of CFD simulations helps to identify the models that need to be used in each region as well as to better explain the results. For example if our simulation conditions are such that the fluid only exists in a gas-like phase, then we could use much simpler linear thermophysical models without losing accuracy.

\section{NUMERICAL SIMULATION SETUP}

A numerical framework suitable for simulations of cryogenic fluids at supercritical environments should have two important characteristics. First, the solver should be able to capture accurately the transition of the jet from subcritical (liquid) to supercritical (gas-like) conditions associated with increase in temperature. This is mostly relevant to the choice of the thermophysical models used and the transport of energy (including diffusive transport of heat) modelled in the solver. Secondly, the solver should be able to simulate accurately both turbulent mixing through either appropriate submodels or adequate grid resolution, as well as the mixing between the injected fluid and the chamber fluid due to mass diffusion, as soon as the fluid transitions to the supercritical state. This is particularly relevant for low turbulence miscible flows where turbulent diffusion might not be high enough to assume that molecular diffusion is negligible.

An additional difficulty in forming numerical frameworks is that at supercritcal pressures the cryogenic fluids rapidly transition across the pseudoboiling point. So the traditional Euler-Lagrangian frameworks usually utilised for the modelling of fuel jets/sprays ${ }^{35}$ are not directly applicable, both due to the absence of surface tension (hence absence of droplets) at supercritical pressures and the limitations of the sub grid scale models that are developed for sub-critical conditions. On the other hand, traditional Euler-Euler VoF methods used to simulate immiscible fluids cannot be used for the entire process since for most part of the jet the assumption of immiscibility does not hold ${ }^{36}$.

\section{A. Governing equations of the solver}

In this work we introduce a novel framework based on the so called "one fluid" approach. The in-house solver 'coolFoam' (based on OpenFOAM ${ }^{37}$ ) is designed for compressible non-isothermal two-fluid simulations where diffusive transport of heat and/or mass are also included. The volume fraction equation includes both surface tension effect and diffusive transport of mass (both molecular and thermo-diffusion), and applies them in the appropriate phases of the fluid. The equations are based on volume fraction quantities which provides flexibility to treat the injected fluid through a hybrid approach based on previously developed VoF methodologies for common fluids ${ }^{38}$ in areas that the surface tension effect is important (liquid phase at temperatures below $T_{c r}$ ), and through a conservative species approach that allows for different fluids to diffuse into each other for the regions with miscible phases (gas and/or supercritical).

The governing conservation equations for volume fraction, momentum and energy are solved for a single effective fluid 
TABLE I. Classification of phases and supercritical pseudo-phases based on thermophysical properties of cryogenic fluids

\begin{tabular}{cccccc}
\hline \hline Thermophysical property & Liquid & Transitional & Dense gas-like & (Ideal) gas-like & Gas \\
\hline Heat Capacity & quadratic variation & Drastic spike and fall & quadratic variation & linear variation & linear variation \\
Density & linear variation & cubic variation & quadratic variation & linear variation & linear variation \\
Viscosity & quadratic variation & drastic variation & almost constant & linear variation & linear variation \\
Thermal conductivity & linear variation & drastic variation & almost constant & linear variation & linear variation \\
Surface tension & present btw immiscible fluids & absent & absent & absent & absent \\
Molecular diffusion & present btw miscible fluids & present btw gas-gas, gas-supercritical and supercritical-supercritical \\
Existence regime & subcritical & supercritical & supercritical & supercritical & subcritical \\
\hline \hline
\end{tabular}

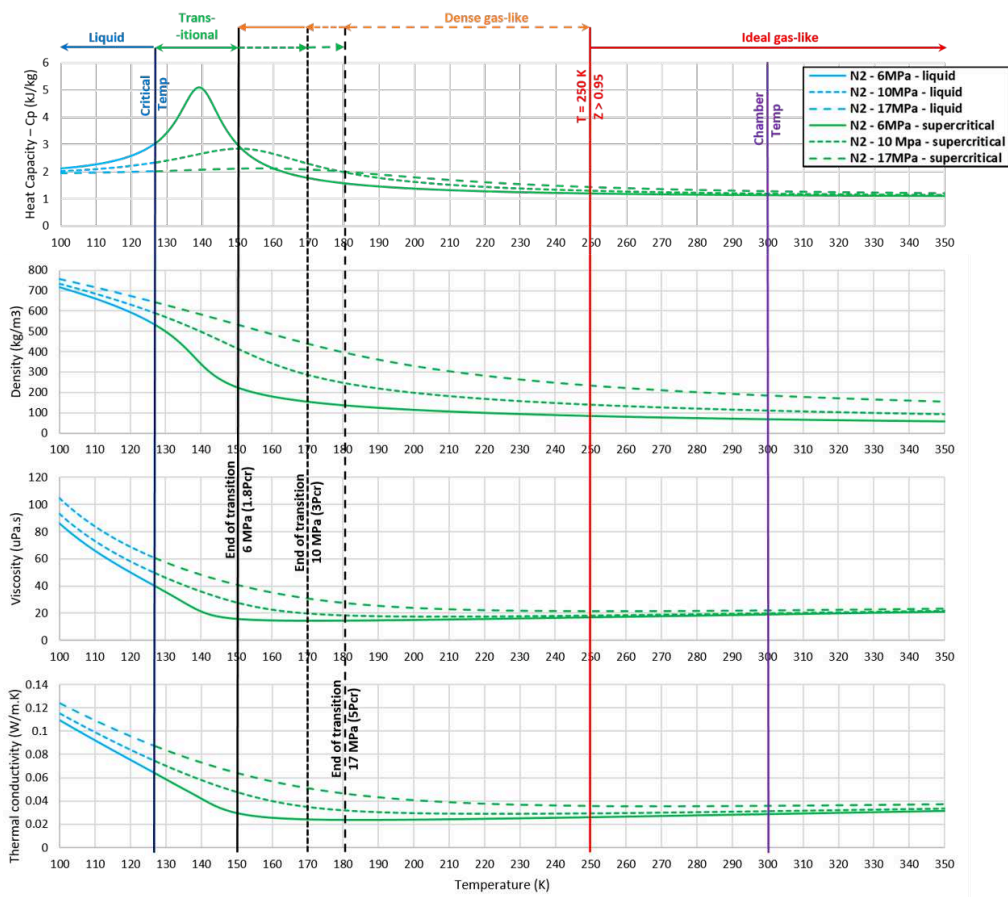

FIG. 2. Classification of phases and supercritical pseudo-phases at supercritical pressures based on the isobaric thermo-physical property (from NIST data) variation of cryogenic nitrogen with temperature. The phase boundaries of the classification are presented for $N_{2}$ at isobaric pressures of $6 \mathrm{MPa}\left(1.8 P_{c r}\right), 10 \mathrm{MPa}\left(3 P_{c r}\right)$ and $17 \mathrm{MPa}\left(5 P_{c r}\right)$.

weighted by the local volume fraction. To begin with, the properties of the single effective fluid are calculated as a weighted mean of each constituent fluid's property, which are obtained through polynomial fitting to NIST (see Sec. III C).
Additional details in brief regarding the 'coolFoam' solver characteristics along with discretisation schemes and thermophysical models utilised is included in Appendix A.

The properties of the single effective fluid is calculated as: 
- Density:

$$
\rho=\rho_{1} \alpha+\rho_{2}(1-\alpha)
$$

- Viscosity:

$$
\mu=\mu_{1} \alpha+\mu_{2}(1-\alpha)
$$

where 1 and 2 refers to the constituent fluids, injection and chamber fluid respectively.

The governing conservation equations are

- Volume fraction equation:

$$
\frac{\partial \alpha}{\partial t}+\nabla \cdot(\mathbf{U} \alpha)-\nabla \cdot\left(D \nabla \alpha+D_{T} \nabla T\right)=\alpha(1-\alpha)\left(\frac{\psi_{2}}{\rho_{2}}-\frac{\psi_{1}}{\rho_{1}}\right) \frac{D p}{D t}+\alpha \nabla \cdot(\mathbf{U})
$$

where $\alpha=\alpha_{1}$ is the volume fraction occupied by fluid $1,(1-\alpha)=\alpha_{2}$ the volume fraction occupied by fluid $2, U$ is the velocity of the single effective fluid, $D$ is the molecular diffusion coefficient, $D_{T}$ is thermo-diffusion coefficient, $p$ is the pressure, $\psi$ is the compressibility and $\rho$ is the density of the fluid. The subscripts ' 2 ' and ' 1 ' correspond to the fluid 2 and fluid 1 respectively.

- Momentum equation:

$$
\frac{\partial \rho \mathbf{U}}{\partial t}+\nabla \cdot(\rho \mathbf{U U})-\nabla \cdot(\mu \nabla \mathbf{U})=\sigma k_{c} \nabla \alpha-\mathbf{g} \cdot \mathbf{x} \nabla \rho-\nabla p_{d}
$$

where $\sigma$ is the surface tension coefficient specified by the user, $k_{c}$ is the curvature of the interface between fluids calculated by the solver as $k_{c}=\nabla \cdot(\nabla \alpha /|\nabla \alpha|), p_{d}$ is the dynamic pressure calculated as $p_{d}=p-\rho \mathbf{g x}$, where $\mathbf{g}$ is the gravity and $\mathbf{x}$ is the position vector.

- Temperature equation (energy):

$\frac{\partial}{\partial t}(\rho T)+\nabla \cdot(\rho U T)-\nabla \cdot\left(k_{e f f} \nabla T\right)-\nabla \cdot\left(\frac{\left(\rho_{1} C_{v, 1}-\rho_{2} C_{v, 2}\right)}{C_{v, 12}}\left(D \nabla \alpha+D_{T} \nabla T\right) T\right)$

$$
=-\frac{1}{C_{v, 12}}\left(\nabla \cdot(P U)+\frac{\partial}{\partial t}(\rho K)+\nabla \cdot(\rho U K)\right)
$$

where $T$ is the temperature, $K$ is the kinetic energy calculated by the solver as $K=\left(|\mathbf{U}|^{2} / 2\right), C_{v, 1}$ and $C_{v, 2}$ are the specific heat capacities at constant volume for fluid 1 and fluid 2 respectively. The term $1 / C_{v, 12}$ is the inverse of heat capacity of the single effective fluid and is calculated as $\left(1 / C_{v, 12}=\alpha_{1} / C_{v, 1}+\alpha_{2} / C_{v, 2}\right)$

\section{B. Simulation cases}

The cases simulated are presented in Table III and the critical properties of nitrogen are presented in Table II. The firs case 'lowVel-2Pc' is matched with Mayer at al's experimental "Case 9"10,39, and is utilised to verify the accuracy of the solver. Three isobaric supercritical pressures $\left(2,3\right.$ and $\left.5 P_{c r}\right)$ and two injection velocities $(2$ and $10 \mathrm{~m} / \mathrm{s})$ are simulated. The injection temperature is just above the critical point $(\mathrm{T}=135 \mathrm{~K}$, $\left.1.1 T_{c} r\right)$ for all the simulated cases, although below the pseudoboiling temperature of nitrogen at all the three pressures. The simulation cases are chosen to analyse the influence of turbulence and diffusion at various Re numbers in a supercritical cryogenic (nitrogen) jet where the fluid transitions across the pseudo-boiling point. It should be pointed out that the
Reynolds variability (from $5 \times 10^{4}$ to $33 \times 10^{4}$ ) is succeeded by altering both the pressure (which in turn alters the thermophysical properties including density and viscosity) as well as the velocity which directly affects the aerodynamic influence on the jet evolution. An additional case of $50 \mathrm{~m} / \mathrm{s}$ has also been simulated corresponding to an extreme of $\mathrm{Re}=125 \times 10^{4}$. The data are not included here to keep the context focused. Comments at the explanation of the presented results are included when necessary to clarify if the observed trends are only limited to a specific range of Re numbers or if they are general for a wider range of cases. 
TABLE II. Critical properties of nitrogen $\left(\mathrm{N}_{2}\right)$.

\begin{tabular}{lccc}
\hline \hline & $\begin{array}{c}\text { Critical } \\
\text { Pressure } \\
(\mathrm{MPa})\end{array}$ & $\begin{array}{c}\text { Critical } \\
\text { Temperature } \\
(\mathrm{K})\end{array}$ & $\begin{array}{c}\text { Critical } \\
\text { Density } \\
\left(\mathrm{kg} / \mathrm{m}^{3}\right)\end{array}$ \\
\hline Nitrogen $\left(\mathrm{N}_{2}\right)$ & 3.39 & 126.2 & 313.3 \\
\hline \hline
\end{tabular}

\section{Thermophysical properties models}

Previous research efforts in numerical simulations of cryogenic jets by various researchers (see 15, 40-45) have demonstrated that the density of cryogenic fluids can be modelled reasonably well through cubic Equations of States (EOS) such as the Peng Robinson (PR) ${ }^{46}$ and the Soave Redlich Kwong $(\mathrm{SRK})^{47}$. The thermodynamic properties can be obtained from thermodynamic derivations from the chosen EOS. The transport properties at high pressures can be defined by additional models used in combination with these EOS, such as the Chung et al's model ${ }^{48}$ for viscosity and thermal conductivity. In addition to these thermophysical properties, recent experimental research by Gerber et al. ${ }^{49}$ and numerical simulations by Foll et al. ${ }^{50}$ has revealed that the diffusive transport of mass cannot be omitted at high pressures (which corresponds to supercritical pressures of cryogenic fluids).

In this work an alternative approach is followed. The thermophysical properties of nitrogen at isobaric supercritical pressures (density, heat capacity, viscosity and thermal conductivity) are modelled through polynomial fitting of NIST for the temperature range $100-350 \mathrm{~K}$. More details about this approach can be found in our previous publications(Ref. 51 and 6) where we have provided detailed discussions of the trend in thermophysical properties of $\mathrm{N}_{2}$ against temperature at various isobaric subcritical and supercritical pressures as well as the performance of various EoS. We also demonstrated that the polynomial fits of density, viscosity and thermal conductivity were equal or more accurate than the PR and SRK predictions. The mass diffusion coefficients $D$ and $D_{T}$ of nitrogen at atmospheric pressure are estimated through models specified in 52 and 53 which are then translated for high pressures using the generalised chart and method described by Takahashi ${ }^{54}$. The mass diffusion coefficient values used in our simulations are presented in Table IV.

\section{Domain, mesh and boundary conditions}

A 3D, axi-symmetric and cylindrical mesh is used, in which the nitrogen is injected through a circular $2.2 \mathrm{~mm}$ diameter inlet at the axis. Pressure outlet is imposed at the the cylinder surface opposite to inlet, All other cylinder surfaces are considered as isothermal walls maintained at chamber temperature $(300 \mathrm{~K})$. First a $2 \mathrm{D}$ plane mesh is generated with the dimensions, discretisation and grading as shown in Fig. 4. The $3 \mathrm{D}$ cylindrical axi-symmetric mesh is obtained by extruding this $2 \mathrm{D}$ mesh around the axis with 32 layers as shown in the figure Fig. 3. The resolution of the axi-symmetric cylindrical mesh increases both towards the injection in axial direction and towards the centreline in the radial direction, ensuring that sufficient resolution is present along the jet boundary.A digital inflow generator ${ }^{55}$ with a turbulent length scale of $\mathscr{L}_{t} \approx 0.04 \mathrm{~d}$ and an inflow with a turbulence intensity of $5 \%$ is used to generate fully developed inlet velocity profile. The fluid's injection temperature is assumed constant at $135 \mathrm{~K}$.

Previous numerical simulations by researchers using RANS and LES have revealed that the common RANS models simulate with almost same accuracy as that of the LES models, and the simulation accuracy depends on the chosen thermophysical model ${ }^{42,43,45}$. We have also performed sensitivity tests of simulation with no turbulence model used and LES with turbulence modelling which have shown no improvement in the time averaged results. Moreover, the LES models used commonly were not developed for cryogenic fluids especially in conditions where thermophysical properties of the fluid vary drastically. Since experimental results of centreline density in cryogenic jets by Mayer et al. ${ }^{10,39}$ are compared against time averaged simulation results and due to the identified discrepancies in the measurement of injection temperature by Mayer et al. ${ }^{10,39}$, it is impossible to identify from previous studies which of the LES models are more fit for cryogenic fluids. For all the above reasons we choose to use a no-turbulence model approach. The grid dependency study (with no turbulence model) is included in Appendix B, where it can be observed that the grid independent solution is close to the mesh used in the simulations 'Simulation mesh'.

\section{RESULTS AND DISCUSSION}

\section{A. Jet evolution}

Instantaneous profiles (at $t=1 \mathrm{~s}$ ) of density, temperature and volume fraction of nitrogen injected at $135 \mathrm{~K}$ into a isobaric chamber maintained at $6 \mathrm{MPa}$ supercritical pressure and $300 \mathrm{~K}$ for two different injection velocities are presented in Fig. 5. The colouring in these contours has been performed based on the absolute values of the properties. The density contour in the figure provides a visualisation of the gradual transition of the fluid from a dense fluid to light gas as the fluid heats up inside the supercritical $\mathrm{N}_{2}$ environment. The heating up process can be better seen in the temperature profile. The volume fraction profiles reflect the mixing effect of the injected fluid and the chamber fluid. Only minor differences are observed between the profiles for the two velocities regardless of the considerably different Re numbers $\left(6.75 \times 10^{4}\right.$ and $33.63 \times 10^{4}$ respectively).

Figure 6 shows the instantaneous profiles at $t=1 \mathrm{~s}$ of the available enthalpy difference (enthalpy difference between the enthalpy of the injected nitrogen and the enthalpy of the nitrogen at chamber temperature) for heat absorption. Contours of temperature developed in the chamber due to continuous injection of $\mathrm{N}_{2}$ up to $1 \mathrm{~s}$, are marked in black. This difference in enthalpy is used to visualise the distribution of the heat absorption potential of a cryogenic jet at various pressures. We can observe that with increase in supercritical pressure the cooler chamber temperature regions decrease. This indicates 
TABLE III. Table of cases of $\mathrm{N}_{2}$ at $135 \mathrm{~K}$ injected through a $2.2 \mathrm{~mm}$ diameter orifice into a chamber filled with gaseous $\mathrm{N}_{2}$ at $300 \mathrm{~K}$ for simulating various supercritical pressure conditions

\begin{tabular}{ccccccc}
\hline \hline & Case & \multicolumn{2}{c}{ Injection } & \multicolumn{2}{c}{ Chamber } & \\
No & Name & Temp $(K)$ & Velocity $(\mathrm{m} / \mathrm{s})$ & Pressure $(M P a)$ & Temp $(K)$ & Experiment \\
\hline 1 & lowVel-2Pc & $135\left(1.1 T_{c r}\right)$ & 2 & $6\left(1.8 P_{c r}\right)$ & $300\left(2.4 T_{c r}\right)$ & Mayer case $9^{10}$ \\
2 & highVel-2Pc & $135\left(1.1 T_{c r}\right)$ & 10 & $6\left(1.8 P_{c r}\right)$ & $300\left(2.4 T_{c r}\right)$ & \\
3 & lowVel-2Pc & $135\left(1.1 T_{c r}\right)$ & 2 & $10\left(3 P_{c r}\right)$ & $300\left(2.4 T_{c r}\right)$ & \\
4 & highVel-3Pc & $135\left(1.1 T_{c r}\right)$ & 10 & $10\left(3 P_{c r}\right)$ & $300\left(2.4 T_{c r}\right)$ \\
5 & lowVel-5Pc & $135\left(1.1 T_{c r}\right)$ & 2 & $17\left(5 P_{c r}\right)$ & $300\left(2.4 T_{c r}\right)$ & \\
6 & highVel-5Pc & $135\left(1.1 T_{c r}\right)$ & 10 & $17\left(5 P_{c r}\right)$ & $300\left(2.4 T_{c r}\right)$ & \\
\hline \hline
\end{tabular}

TABLE IV. Mass diffusion coefficients associated with the simulation cases

\begin{tabular}{|c|c|c|c|c|}
\hline \multirow{2}{*}{$\begin{array}{l}\text { Simulation } \\
\text { case }\end{array}$} & \multirow{2}{*}{$\begin{array}{c}\text { Pressure } \\
(\mathrm{MPa})\end{array}$} & \multirow{2}{*}{$\begin{array}{c}\text { Temperature } \\
(K)\end{array}$} & \multicolumn{2}{|c|}{ Diffusion coefficient } \\
\hline & & & molecular - $D\left(\mathrm{~m}^{2} / \mathrm{s}\right)$ & soret/thermo - $D_{T}\left(m^{2} / K s\right)$ \\
\hline lowVel/highVel-2Pc & $6\left(1.8 P_{c r}\right)$ & $300\left(2.4 T_{c r}\right)$ & $3.3 \times 10^{-7}$ & $0.15 \mathrm{D}$ \\
\hline lowVel/highVel-3Pc & $10\left(3 P_{c r}\right)$ & $300\left(2.4 T_{c r}\right)$ & $2 \times 10^{-7}$ & $0.15 \mathrm{D}$ \\
\hline lowVel/highVel-5Pc & $17\left(5 P_{c r}\right)$ & $300\left(2.4 T_{c r}\right)$ & $1.18 \times 10^{-7}$ & $0.15 \mathrm{D}$ \\
\hline
\end{tabular}

\begin{tabular}{|c|c|c|c|c|}
\hline & $\begin{array}{l}\text { Min cell vol } \\
\left(\mathrm{m}^{3}\right)\end{array}$ & $\begin{array}{l}\text { Max cell vol } \\
\left(\mathrm{m}^{3}\right)\end{array}$ & $\begin{array}{c}\sqrt[3]{(\text { Mincellvol })} \\
(\mathrm{m})\end{array}$ & $\begin{array}{c}\sqrt[3]{\text { Maxcellvol }} \\
(\mathrm{m})\end{array}$ \\
\hline Mesh & $1.953 \times 10^{-12}$ & $2.606 \times 10^{-7}$ & $1.250 \times 10^{-4}$ & $6.388 \times 10^{-3}$ \\
\hline
\end{tabular}

that the heat absorption potential of cryogenic fluids decreases with increase in the chamber supercritical pressure, which is associated with the very low heat capacity and distributed latent heat ${ }^{51}$ at higher supercritical pressures.

According to the classification of phases and pseudophases we have introduced in Section II, the simulation results of instantaneous $(t=1 s)$ and time averaged jet profiles are presented in Fig. 7 for all simulated cases. It can be seen that the transitional phase is encapsulated within a supercritical dense gas phase which in turn is encapsulated within the supercritical gas phase. An important finding is that regardless of the different injection velocities $(2$ or $10 \mathrm{~m} / \mathrm{s}$ ) and Re numbers, the jets profiles develop very similarly, suggesting that the thermodynamic mechanism plays a more dominant role in the jet evolution at these pressures rather than the aerodynamic one. With increase in supercritical pressure, the cryogenic fluid transitions rapidly from liquid/liquid-like to (ideal) gas-like, as the pseudoboiling effect diminishes.

\section{B. Centerline Properties Variation}

The time averaged axial centreline distribution of density and temperature for all the simulations are presented in Fig. 8. The centreline density distribution represents the jet decay/breakup whereas the centreline temperature distribution provides a better visualisation of how this process is associated with the jet heating up process.

The centreline density distribution from the simulated case
'lowVel-2Pc' agrees well with the experimental measurements of nitrogen jet injected in identical conditions by Mayer et al. ${ }^{10}$ from $x / d=10$ to 30 . Some discrepancies are observed up to $x / d=10$. The same trend is also observed in previous numerical simulations by other researchers ${ }^{41-43,45,56}$. Banuti $^{15}$ has stated that the adiabatic assumption of the injector in numerical simulations results in the density at injection being constant for some axial distance along the centreline. In contrast, in the experiment by Mayer et al. ${ }^{10}$ the density drops immediately upon entering the chamber. This is because, in the case of adiabatic injector the heat transfer to injected fluid starts only after the fluid enters the chamber. Mayer et al. ${ }^{10}$ recorded different temperature measurements at the core and boundary of the nitrogen jet at the location of the injection, supporting the argument for non-adiabatic injection conditions. Since it is challenging to quantify the heat transfer due to the uncertainty in the measured boundary temperature in our numerical simulations we use a uniform injection temperature at the inlet which recreates the same inlet temperature profile as that of an adiabatic injector and explains the initial deviation.

Based on the above figure, it can be seen that supercritical $\mathrm{N}_{2}$ jets possess three distinct zones similar to other subcritical fluid jets: potential core up to approximately $x / d=5$, transitional region, up to $x / d=20$ and self-similar for the remaining of the jet. With increase in pressure the jet breakup occurs earlier (and the potential core reduces). Increase in supercritical pressures also results in a quicker decay of the jet, which can be observed from the steeper slopes of density (drop) and temperature (rise) at higher supercritical pressures. One important difference though with other subcritical jets is that at the transitional zone of these supercritical jets the jet decays both due to the mixing with the much less dense gas found in the chamber $\left(\mathrm{N}_{2}\right.$ at $\left.300 \mathrm{~K}\right)$, as well as due to the heat up of the fluid which as time progresses reduces further the density. A more quantitative analysis of the potential core as well as similarities and differences with sub critical fluid jets will be 

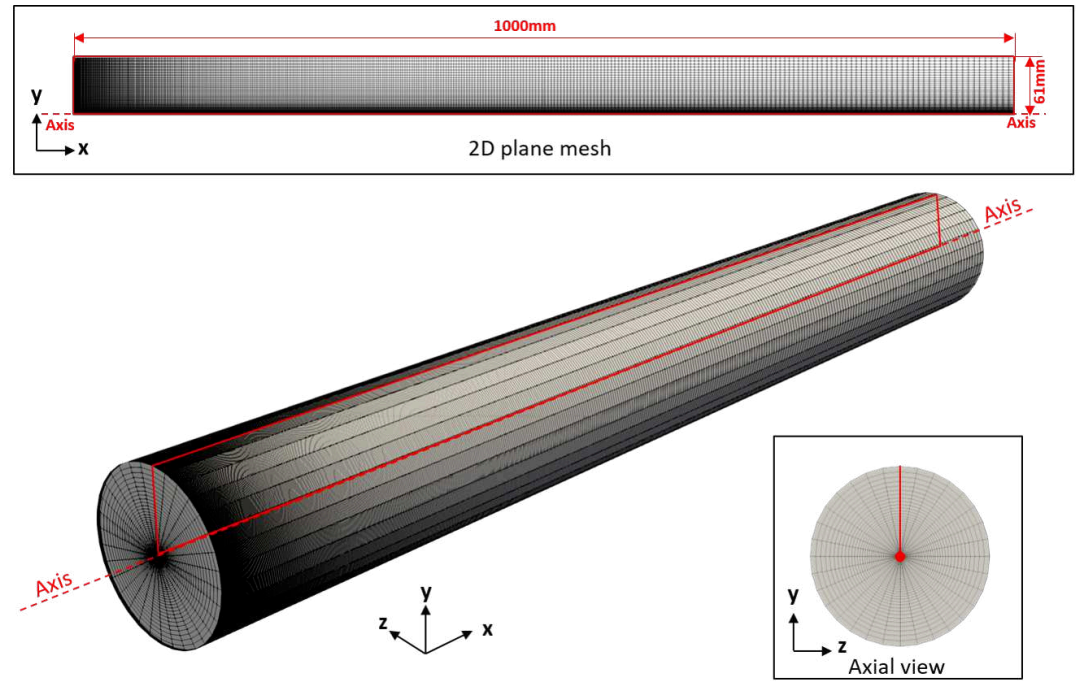

FIG. 3. 3D cylindrical axi-symmetric mesh created from 2D plane mesh

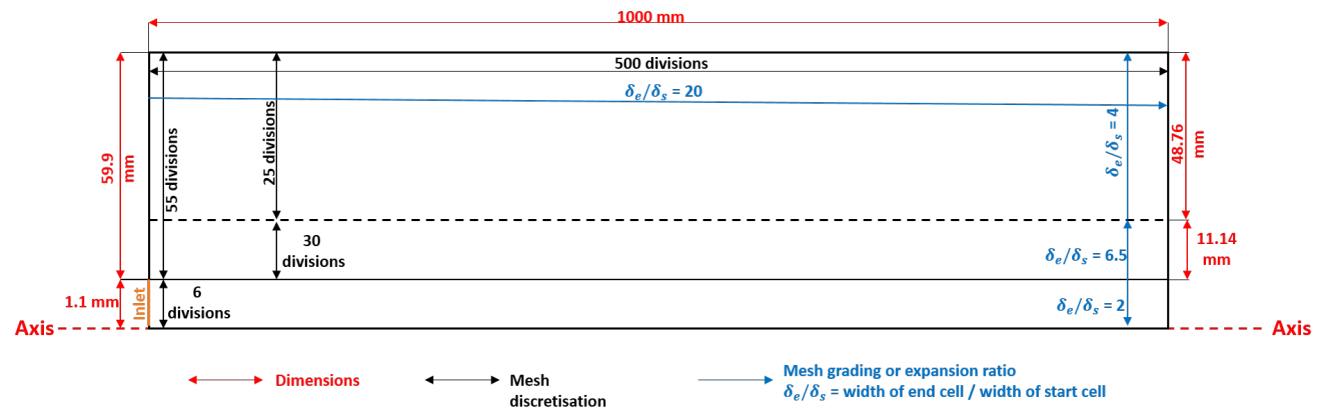

FIG. 4. Dimensional, discretisation and grading details of the 2D plane mesh domain

TABLE VI. Table of injection conditions and associated turbulence scales.

\begin{tabular}{|c|c|c|c|c|c|c|c|c|c|}
\hline $\begin{array}{l}\text { Case } \\
\text { name }\end{array}$ & $\begin{array}{c}\text { Dia - } 1_{\text {ref }} \\
(m)\end{array}$ & $\begin{array}{c}\text { vel } \\
(\mathrm{m} / \mathrm{s}) \\
\end{array}$ & $\begin{array}{c}\text { Pressure } \\
(M P a)\end{array}$ & $\begin{array}{c}\text { Temp } \\
(K)\end{array}$ & $\begin{array}{l}\text { Density } \\
\left(\mathrm{kg} / \mathrm{m}^{3}\right)\end{array}$ & $\begin{array}{c}\text { Viscosity } \\
(\text { Pa.s })\end{array}$ & $\begin{array}{c}\text { Reynolds no } \\
(R e)\end{array}$ & $\begin{array}{c}\text { Taylor } \\
\text { scale }\left(\tau / l_{r e f}\right)\end{array}$ & $\begin{array}{l}\text { Kolmogorov } \\
\text { scale }\left(\kappa / l_{\text {ref }}\right)\end{array}$ \\
\hline lowVel-2Pc & 0.0022 & 2 & $6\left(1.8 P_{c r}\right)$ & $135\left(1.07 T_{c r}\right)$ & 430.05 & 28.132 & $6.75 \times 10^{4}$ & $1.27 \times 10^{-2}$ & $2.388 \times 10^{-4}$ \\
\hline highVel-2Pc & 0.0022 & 10 & $6\left(1.8 P_{c r}\right)$ & $135\left(1.07 T_{c r}\right)$ & 430.05 & 28.132 & $33.63 \times 10^{4}$ & $0.55 \times 10^{-2}$ & $0.72 \times 10^{-4}$ \\
\hline lowVel-3Pc & 0.0022 & 2 & $10\left(3 P_{c r}\right)$ & $135\left(1.07 T_{c r}\right)$ & 537.19 & 40.731 & $5.80 \times 10^{4}$ & $1.313 \times 10^{-2}$ & $2.676 \times 10^{-4}$ \\
\hline highVel-3Pc & 0.0022 & 10 & $10\left(3 P_{c r}\right)$ & $135\left(1.07 T_{c r}\right)$ & 537.19 & 40.731 & $29.02 \times 10^{4}$ & $0.59 \times 10^{-2}$ & $0.80 \times 10^{-4}$ \\
\hline lowVel-5Pc & 0.0022 & 2 & $17\left(5 P_{c r}\right)$ & $135\left(1.07 T_{c r}\right)$ & 606.74 & 52.561 & $5.08 \times 10^{4}$ & $1.40 \times 10^{-2}$ & $2.96 \times 10^{-4}$ \\
\hline highVel-5Pc & 0.0022 & 10 & $17\left(5 P_{c r}\right)$ & $135\left(1.07 T_{c r}\right)$ & 606.74 & 52.561 & $25.40 \times 10^{4}$ & $0.63 \times 10^{-2}$ & $0.88 \times 10^{-4}$ \\
\hline
\end{tabular}




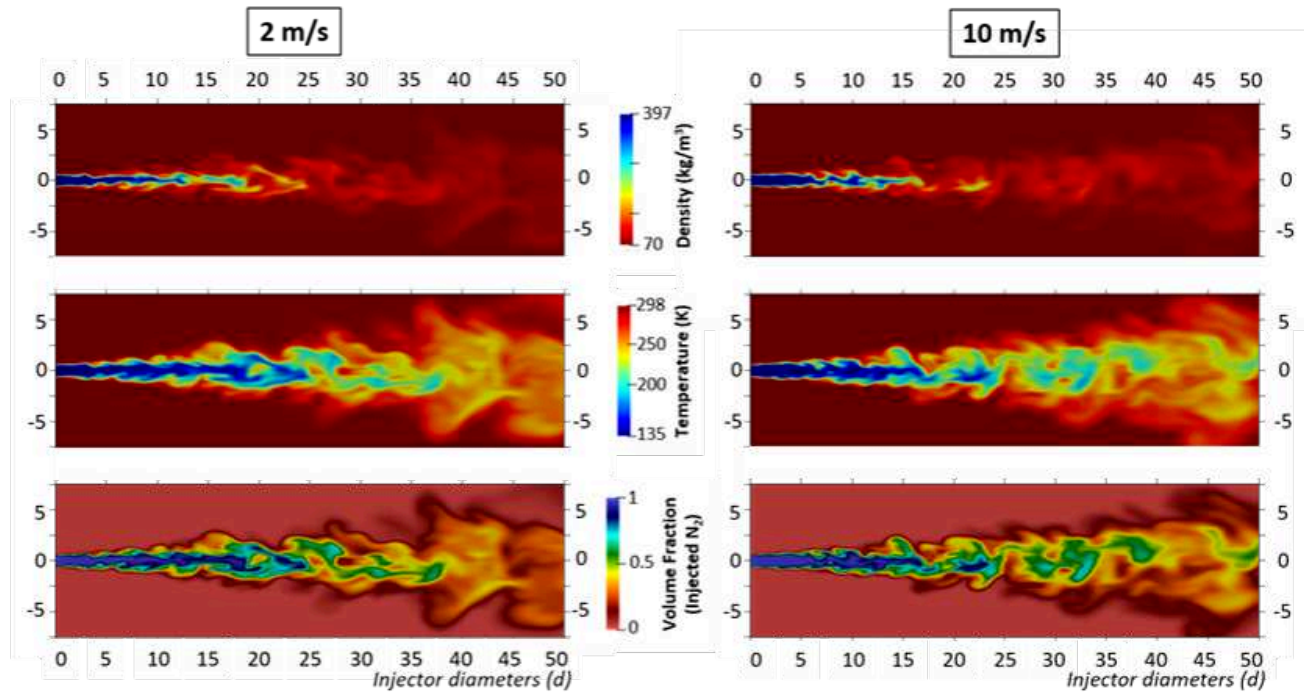

FIG. 5. Instantaneous profiles of density (top), temperature (middle) and volume fraction (bottom) of nitrogen injected at $135 \mathrm{~K}$ with a injection velocity of $2 \mathrm{~m} / \mathrm{s}$ (left) and $10 \mathrm{~m} / \mathrm{s}$ (right).

presented in the next section.

Complementary to Fig. 8 we also include Fig. 9 which shows the non-dimensional centreline density distribution (calculated as suggested by Mayer et al. ${ }^{10}$ ) along with the compressibility factor. For all cases, very little difference in the decay is noticed which further suggests a) the dominance of the thermal (driven) transition over mechanical disintegration for the cases investigated regardless of their very different Re numbers and b) the existence of a rather similar thermal(driven) transition regardless of the supercritical pressure and inlet velocity, provided the injection temperature and chamber temperature are fixed. The compressibility factor increases more rapidly with temperature at lower pressures. It can be seen in the figure that the increase of the compressibility factor is linked to the rapid density decrease downstream of the potential core. After $x / d=25$ the fluid behaves as an ideal gas. We have also ran a supplementary simulation of the $\mathrm{LN}_{2}$ injection at $17 \mathrm{MPa}$ and $50 \mathrm{~m} / \mathrm{s}$. The centreline profiles coincide exactly with the highVel-5PC case (17 MPa and 10 $\mathrm{m} / \mathrm{s}$ ). This further confirms the thermodynamic dominance over mechanical disintegration.

\section{Centreline flow inertia characterisation}

Moving on to the flow characteristics of the jet, we analyse the mean velocity decay, Re number, Root Mean Square (rms) velocities (axial and radial) and relative intensity along the centreline.

Fig. 10 presents the non-dimensional centreline velocity and Re decay side by side for all the cases. The velocity decay is very similar, independent of the initial injection velocity, and is only affected by pressure. The Re decay is steeper than the velocity decay. This is due to the fact that in the case of supercritical jets (in contrast to subcritical jets) Re are affected both by inertia as well as the fluid properties that in our case vary due to the rapidly reducing density as the fluid heats up across the pseudo-boiling temperature.

Figure 11 presents the rms velocities non-dimensionalised with respect to the injection velocity. The centreline distribution of axial rms velocities of 'lowVel' and 'highVel' cases coincide, which leads to the hypothesis that the flow disturbances develop identically in both these 'lowVel' and 'highVel' cases. In all the simulated cases, following an initial suppression of axial and radial rms velocities near the injection, the axial rms velocities develop more rapidly than the radial rms velocities. As we will see in the next section this depends on the density gradients developed. With increase in the supercritical pressures, both the axial and radial velocity fluctuations develop more steeply and peak earlier.

The relative intensity of axial fluctuations with respect to radial fluctuations is presented in Fig. 12. Cryogenic jets do not reach isotropy up to $50 D_{i n j}$ (range of our analysis), due to the persistence of axial fluctuations dominance over radial fluctuations downstream. 
$2 \mathrm{~m} / \mathrm{s}$
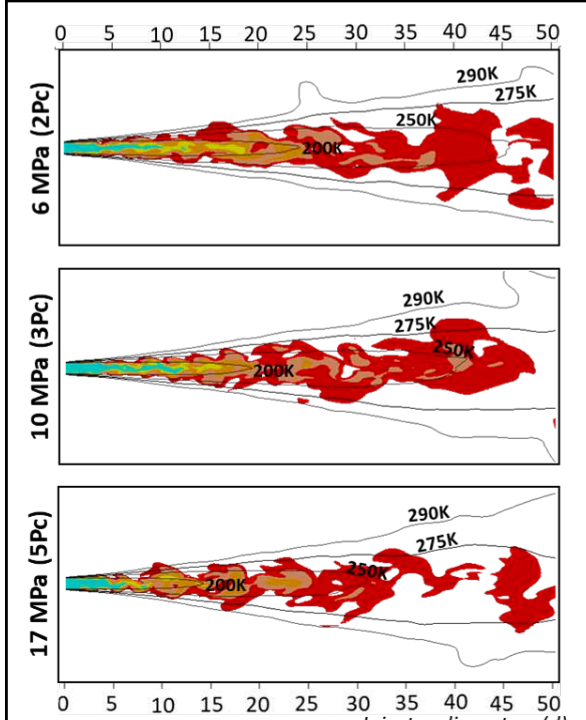

$\begin{array}{lllllllllll}0 & 5 & 10 & 15 & 20 & 25 & 30 & 35 & 40 & 45 & 50 \\ \text { Injector diameters (d) }\end{array}$ $\Delta H_{\text {available }}=\square 50-100 \mathrm{~kJ} / \mathrm{kg}$
$10 \mathrm{~m} / \mathrm{s}$
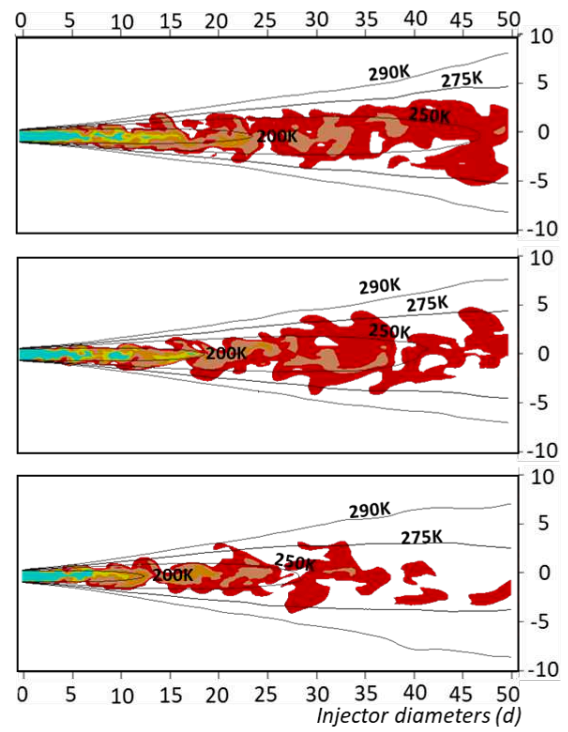

FIG. 6. Instantaneous profiles $(t=1 s)$ of available enthalpy difference (btw the injected $\mathrm{N}_{2}$ and chamber $\mathrm{N}_{2}$ ) for heat absorption in a developed supercritical $N_{2}$ jet. Contours of mean temperature developed in the chamber due to continuous injection of $N_{2}$ up to $1 s$, is marked in black. Injection temperature of $\mathrm{N}_{2}$ is $135 K$ and chamber is initially filled with $300 K \mathrm{~N}_{2}$.

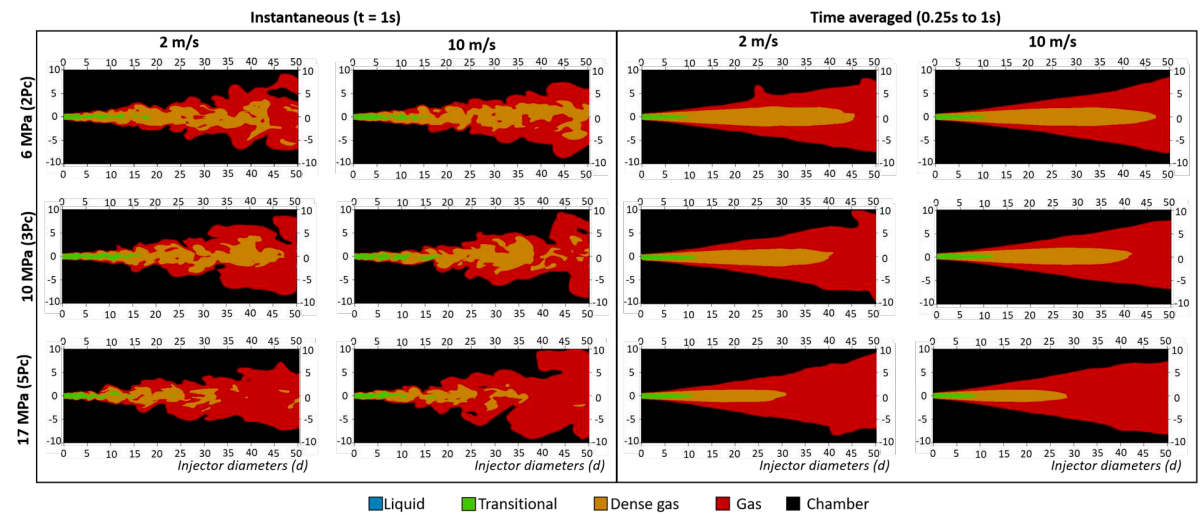

FIG. 7. Instantaneous and mean profiles of pseudophases of $\mathrm{N}_{2}$ injected at $135 \mathrm{~K}$ at various injection velocities and supercritical chamber pressures. 

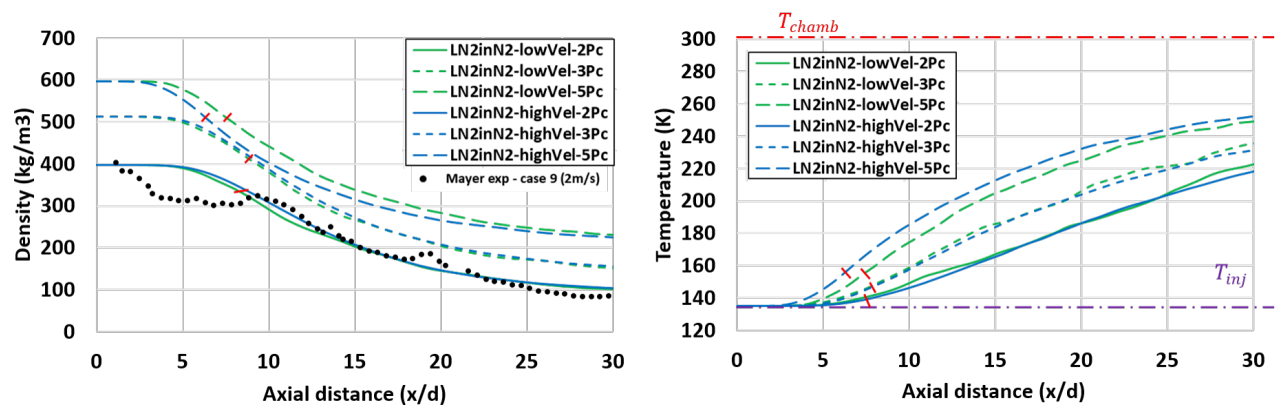

FIG. 8. Axial centreline distribution of density (left) and temperature (right) for the cases simulated. Mayer's case 9 experimental density measurements which corresponds to our case 'lowVel-2Pc' is also plotted. The location where the centreline density and temperature reaches the pseudoboiling values (corresponding to the respective pressure) is marked as red dashes

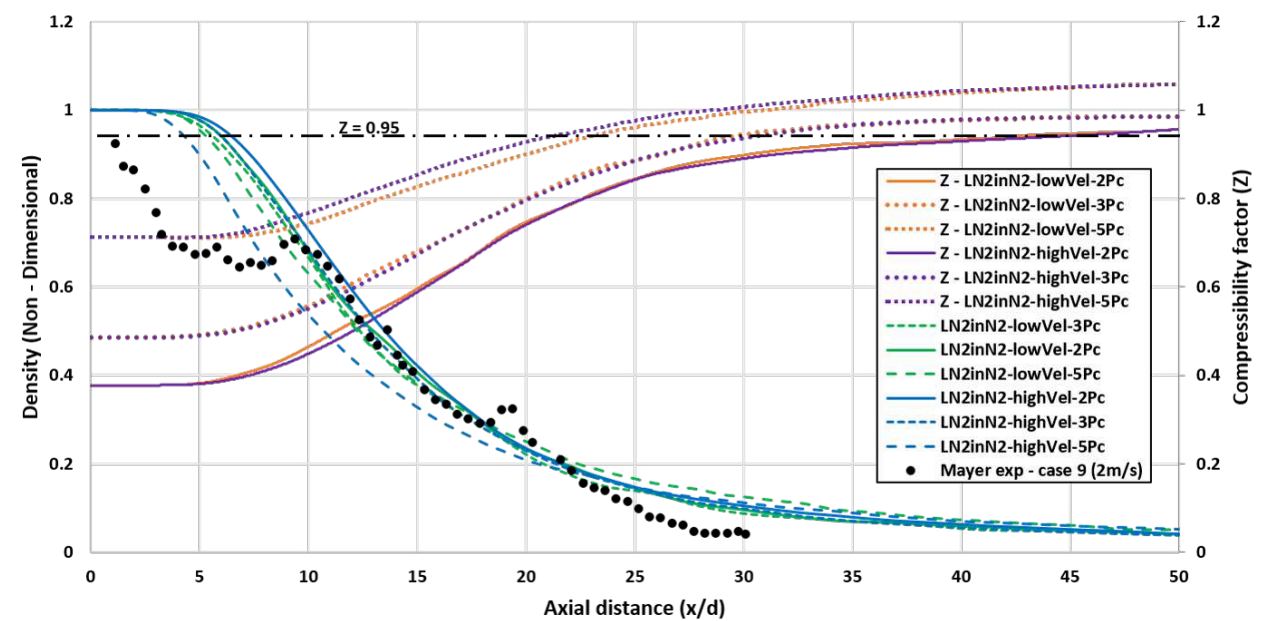

FIG. 9. Non-dimensionalised density distribution along the centreline in the axial direction. Mayer's case 9 experimental density measurements are also non-dimensionalised. The compressibility factor $(\mathrm{Z})$ of the jet along centreline is also plotted in parallel with secondary vertical axis on right.

\section{Density gradients and suppression of turbulence}

Researchers experimenting on supercritical cryogenic jets have long known that the large density gradients at supercritical pressures pose a significant hurdle to optical diagnostic methods, see for example 57. However, the role of density gradients on turbulence development in the jet was not explored in detail in these initial works. Early numerical simulations of cryogenic fluids by Zong et al. ${ }^{40}$ revealed the strong density stratification effects around the jet (near the injection) which act like solid boundaries and dampen the radial oscilla- tions while amplifying the axial oscillations. Later, numerical simulations by other researchers have also confirmed the density stratification effects ${ }^{42-45}$. In the work of Li et al ${ }^{56}$ there is further analysis of the influence of the injection temperature on the density gradient development. From the numerical simulations the authors concluded that the density gradients are stronger and mixing is delayed when the injected fluid transitions from sub/trans-critical to super-critical through the pseudoboiling temperature, as opposed to injection at temperatures above the pseudoboiling temperature. In these previous studies the effect of the initial inertia of the jet was not investigated 

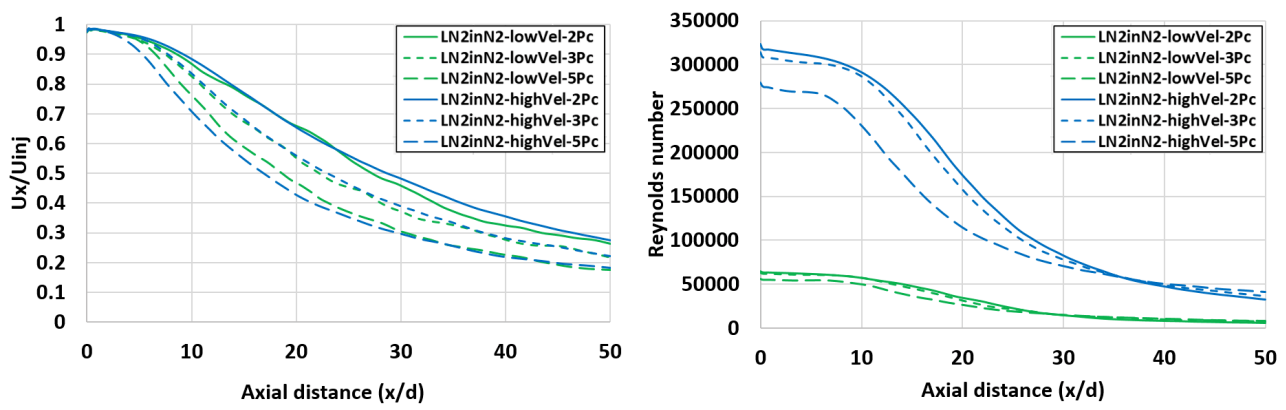

FIG. 10. Time averaged velocity decay (left) and Reynold's number (right) distribution along the centreline for all simulated cases
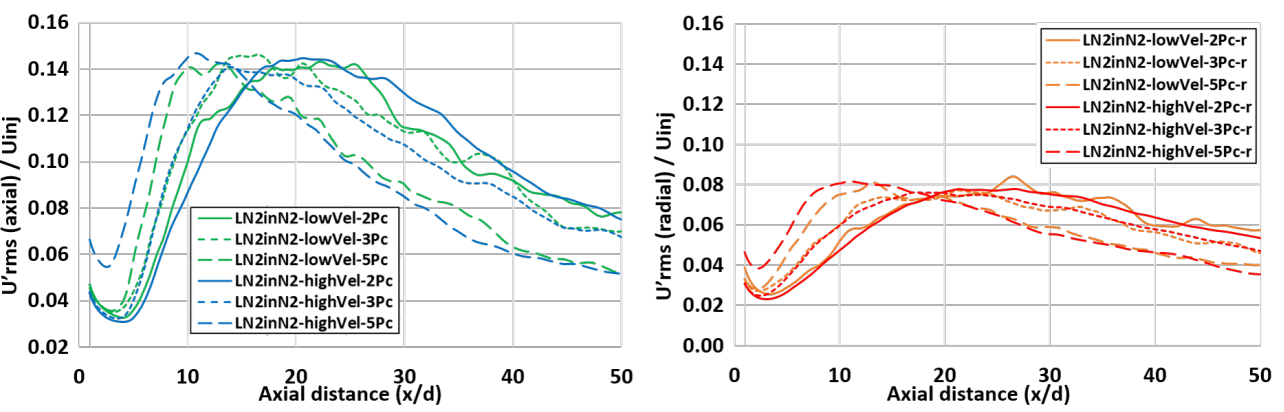

FIG. 11. Time averaged axial (left) and radial (right) rms velocities of all cases non dimensionalised with their respective injection velocity.

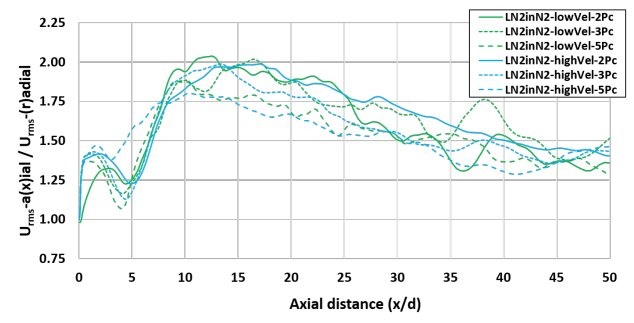

FIG. 12. Relative intensity of axial fluctuations with respect to radial fluctuation

In our work, in order to shed light to the link between coherent structures observed in the instantaneous plots and statistical quantities discussed in the previous sections we include Fig. 13 which visualises where vorticity is located with respect to density gradients. The figure shows the vorticity contour along with the boundary of the strong density gradients ( $>$ order $10^{5}$, black line). It can be observed that the vorticity follows the strong density gradients, with higher magnitude closer to the jet and reduction further downstream.
Figure 14 and Fig. 15 present the density gradients of the highest order $\left(>10^{5}\right)$. The instantaneous 3D structures of these highest order density gradients resemble a hollow tube starting right at the injection point. Close to the injection point a string of strong density-gradient regimes is generated around the jet surface and exerts a stabilising effect on the flow development. Further downstream instabilities develop leading to the collapse of the tube. The instabilities emerge earlier as the pressure increases. For the case of $P=5 P_{c r}$ and $u=10 \mathrm{~m} / \mathrm{s}$ the instabilities appear immediately at the jet exit.

Looking at the cross section of these density gradient tubes at various axial locations, it can be seen that the tube like structures collapse at $x / d=3.75$ to $x / d=5$, for the higher pressure and a bit later for the lower pressure $(x / d=5$ to $x / d=7.5)$. These locations correspond to the location where the time averaged axial and radial rms velocities start to grow significantly. This gives further insight into the suppression effect of these gradients to both the axial and radial fluctuations by confining the flow acts like a solid tube until their structure collapses. After the structure collapses, the axial rms velocity fluctuations increase more rapidly than radial fluctuations. The reason is that even after the collapse of the structure the remaining density gradients are stronger (higher magnitude) in the radial direction than the axial direction and keep 


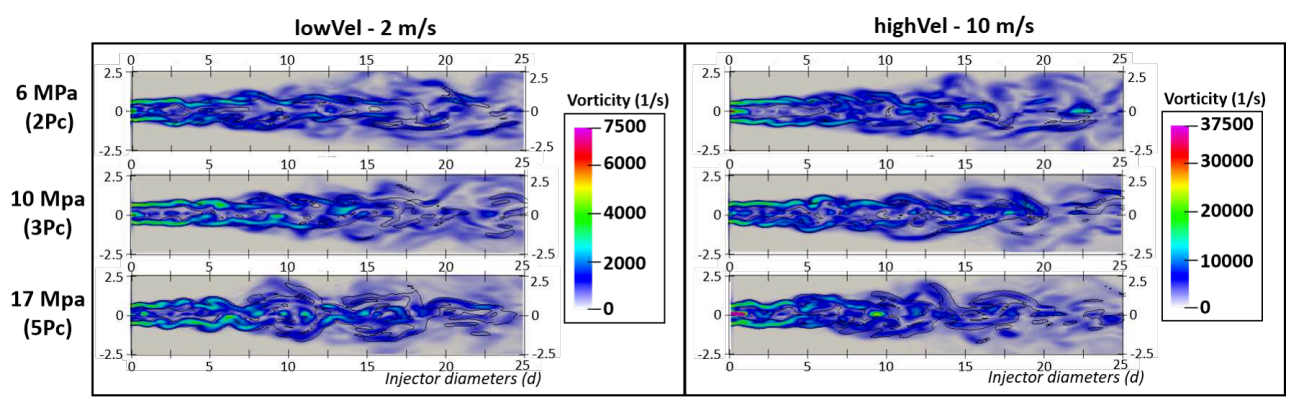

FIG. 13. Vorticity distribution for all cases. The boundary of the strong density gradients $\left(>\right.$ order $\left.10^{5}\right)$ is marked in black.

suppressing radial fluctuations of the velocity.

An important point that needs to be clarified (and has not been discussed in previous studies) is that in the case of supercritical cryogenic jets, these density gradient tubes can arise both due to the thermophysical change in the fluid as well as from the mixing of fluids with different densities. From thermophysical point of view, as the injected fluid heats up, the density starts to drop, this results in higher density at the core which then decreases continuously in the radial direction. This variation in density generates density gradients. A the same time if a heavier fluid is injected into a lighter fluid and the two fluids are miscible, density stratification is also generated. The mass/volume fraction of the injected fluid is maximum at the centreline and decrease radially due to mixing with the ambient fluid. In our cases of supercritical $\mathrm{N}_{2}$ jets, even if the injected and chamber fluid are the same, the temperature difference between the injected fluid and chamber fluid makes them behave as fluids with different densities. In our case of cold $\mathrm{N}_{2}$ injected at $135 \mathrm{~K}$ into the warm $\mathrm{N}_{2}$ at $300 \mathrm{~K}$, the density gradients arise both due to the combined thermophysical transition and mixing up with the chamber $\mathrm{N}_{2}$. This can be observed further in Fig. 16 where the axial cross section of strongest density gradients are presented. The volume fraction varies from maximum to minimum and the temperature varies from minimum to maximum in the radial direction in these cross-sections. Both of these effects add up as both high volume fraction and low temperature results in high density. Also the same effect is evident from Fig. 13 where further downstream although the density gradient tubes has collapsed towards the center, the vorticity at the side of the lighter fluid still exists. At this area, vorticity is mostly result of jet dynamics rather than thermophysics.

\section{E. Density gradients and diffusive mass transport}

Figure 17 presents the contour of the strongest order of density gradients overlapped with the iso-lines of the strongest order of mass flux due to molecular diffusion and thermo (Soret)-diffusion for all the cases. The mass flux due to molecular diffusion ' $D \nabla \alpha$ ' (self diffusion in our cases) de- pends directly on the volume fraction gradient while the thermo(Soret)-diffusion ' $D_{T} \nabla T$ ' depends on the temperature gradient.

It can be observed in Fig. 17 that close to the nozzle, regions of strongest order density gradients co-exist with regions of high molecular diffusion and thermo(Soret)-diffusion. This reveals that the strong density gradients include both the gradient in density due to mixing (volume fraction gradient) and gradient in density due to thermophysical variation with temperature. Further downstream strong density gradients are absent, even though there are regions of high molecular diffusion (strong volume fraction gradients). The reason is that the injected $\mathrm{N}_{2}$, although has not yet been mixed completely with the surrounded chamber gas, has transitioned to a state closer to the state of the chamber $\mathrm{N}_{2}$ with similar densities. As the pressure increases, the density gradients coincide more with the volume fraction gradients and temperature gradients indicating that injected $\mathrm{N}_{2}$ transitions quickly to a state closer to chamber $\mathrm{N}_{2}$. Although the temperature gradient responsible for thermo(Soret)-diffusion does not directly generate density gradients, the thermophysical(density) variation associated with the temperature of the fluid variation results in density gradients.

It is also necessary to point out that although diffusion due to Soret effect is significantly lower than molecular (self) diffusion at pressure, temperature and injection conditions prevailing in our supercritical injection cases (see Fig. 17 and Table VII), it is not entirely negligible. Further, at conditions relevant to combustion chamber of IC and cryogenic rocket engines, the chamber temperature can reach up to $3000 \mathrm{~K}$, which is ten times of the chamber temperature in our simulations, while the cryogenic injection temperature is approximately the same. This results in the temperature gradient ' $\nabla T$ ' being much stronger and hence Soret diffusion can in fact dominate over molecular (self) diffusion in such situations. Thus, it is important to be included in the numerical frameworks. To our knowledge this is the first study that these effects have been accounted for numerically. 


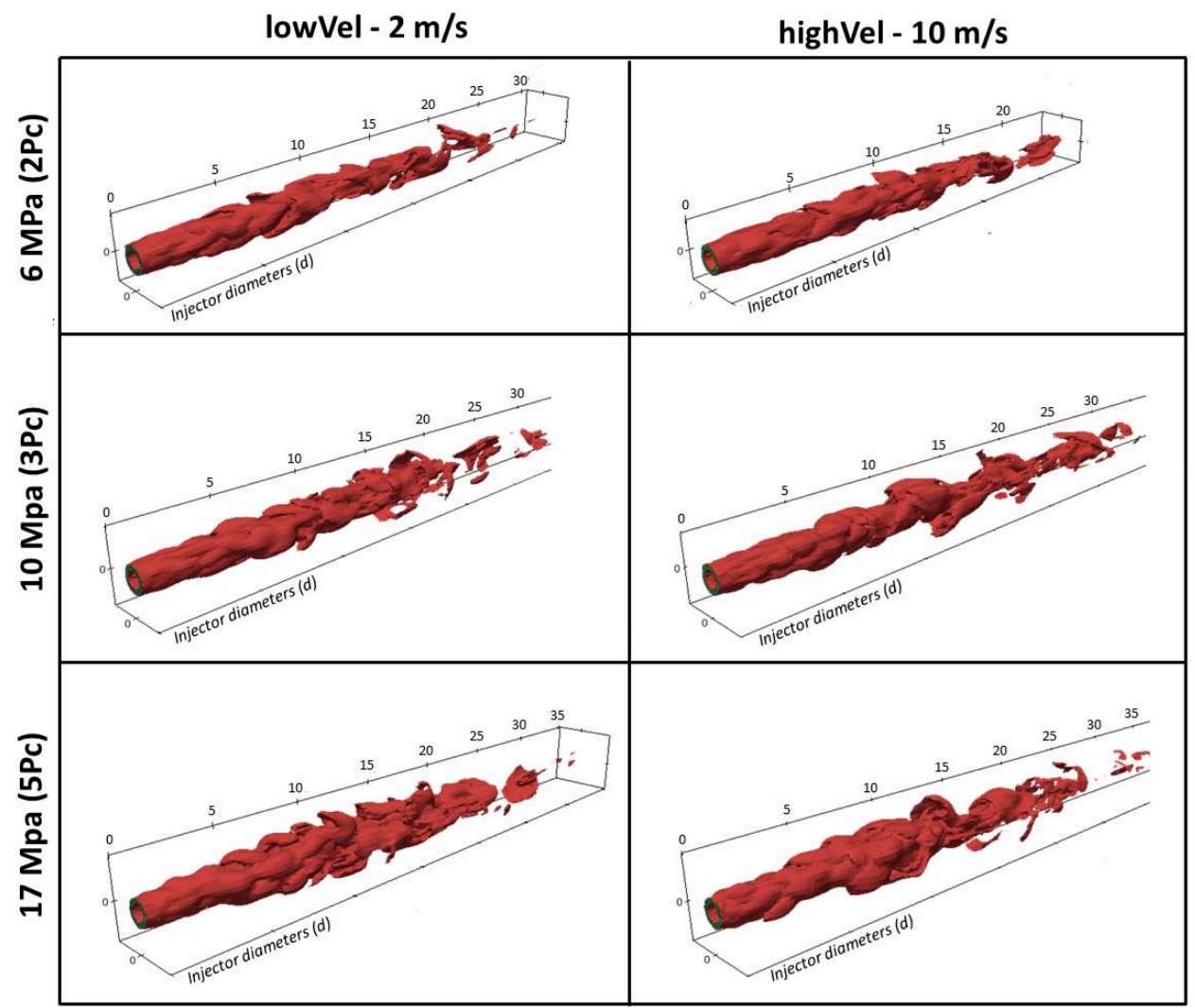

FIG. 14. 3D view of strong density gradients ( $>$ order of $10^{5}$ ) for all cases.

TABLE VII. Magnitude of molecular and thermo (Soret) diffusion flux contours shown in Fig. 17

\begin{tabular}{ccccc}
\hline \hline & Pressure & Temperature & \multicolumn{2}{c}{ Diffusion flux $(m / s)$} \\
Simulation case & $(M P a)$ & $(K)$ & molecular $(D \nabla \alpha)$ & $>1 \times 10^{-4}$ \\
\hline lowVel/highVel-2Pc & $6\left(1.8 P_{c r}\right)$ & $300\left(2.4 T_{c r}\right)$ & $>6 \times 10^{-5}$ & $\left.>1 D_{T} \nabla T\right)$ \\
lowVel/highVel-3Pc & $10\left(3 P_{c r}\right)$ & $300\left(2.4 T_{c r}\right)$ & $>4 \times 10^{-5}$ & $>6 \times 10^{-5}$ \\
lowVel/highVel-5Pc & $17\left(5 P_{c r}\right)$ & $300\left(2.4 T_{c r}\right)$ & & $>6 \times 10^{-6}$ \\
\hline \hline
\end{tabular}

\section{DISCUSSION ON THE BEHAVIOUR OF SUPERCRITICALL CRYOGENIC JETS IN COMPARISON TO OTHER FLUID JETS}

In subcritical turbulent jets (including cryogenic jets) commonly three distinct areas are identified in the literature: the potential core, the transitional region and the fully developed self similar region ${ }^{10}$. Looking at the mean contours of our supercritical cryogenic jets presented in Fig. 7 as well as the den- sity profiles in Fig. 9 we can also identify three areas which however are separated based on pseudo phases rather than the inertia characteristic of the jet. The question that arises -and we will address here- is if these areas based on thermophysics also have some distinct mechanical characteristics that resemble other common liquid and gas jets at sub-critical conditions.

The potential core is the near-field region that the fluid and its properties are identical to the ones at injection. In turbu- 

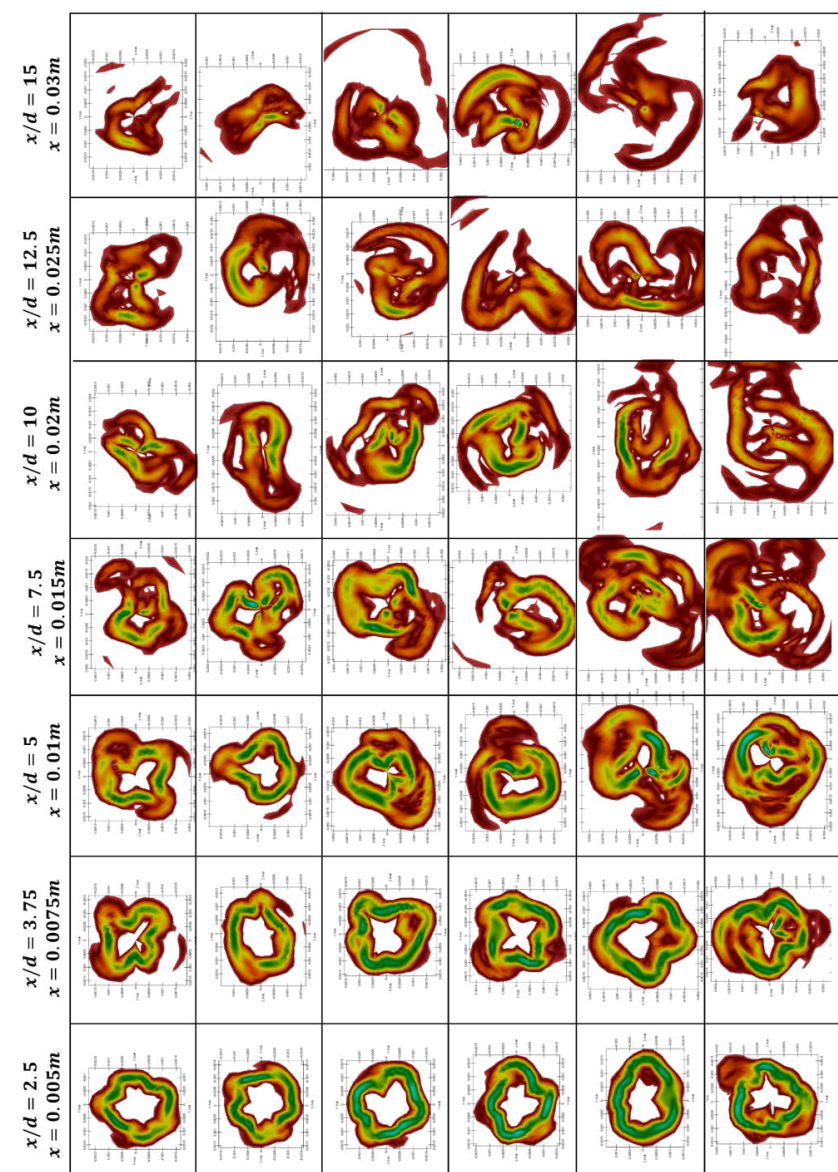

空
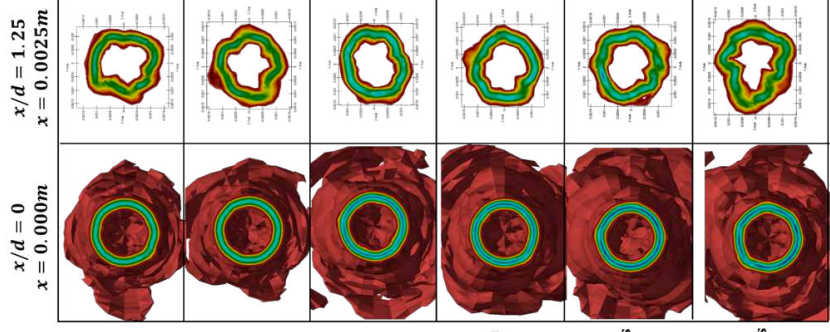

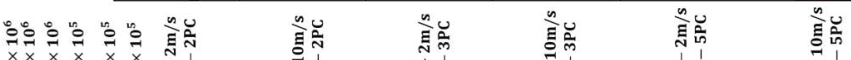

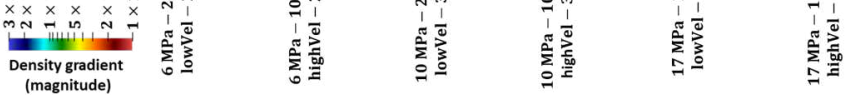

FIG. 15. Radial cross section of the density gradient tubes at various axial locations. 


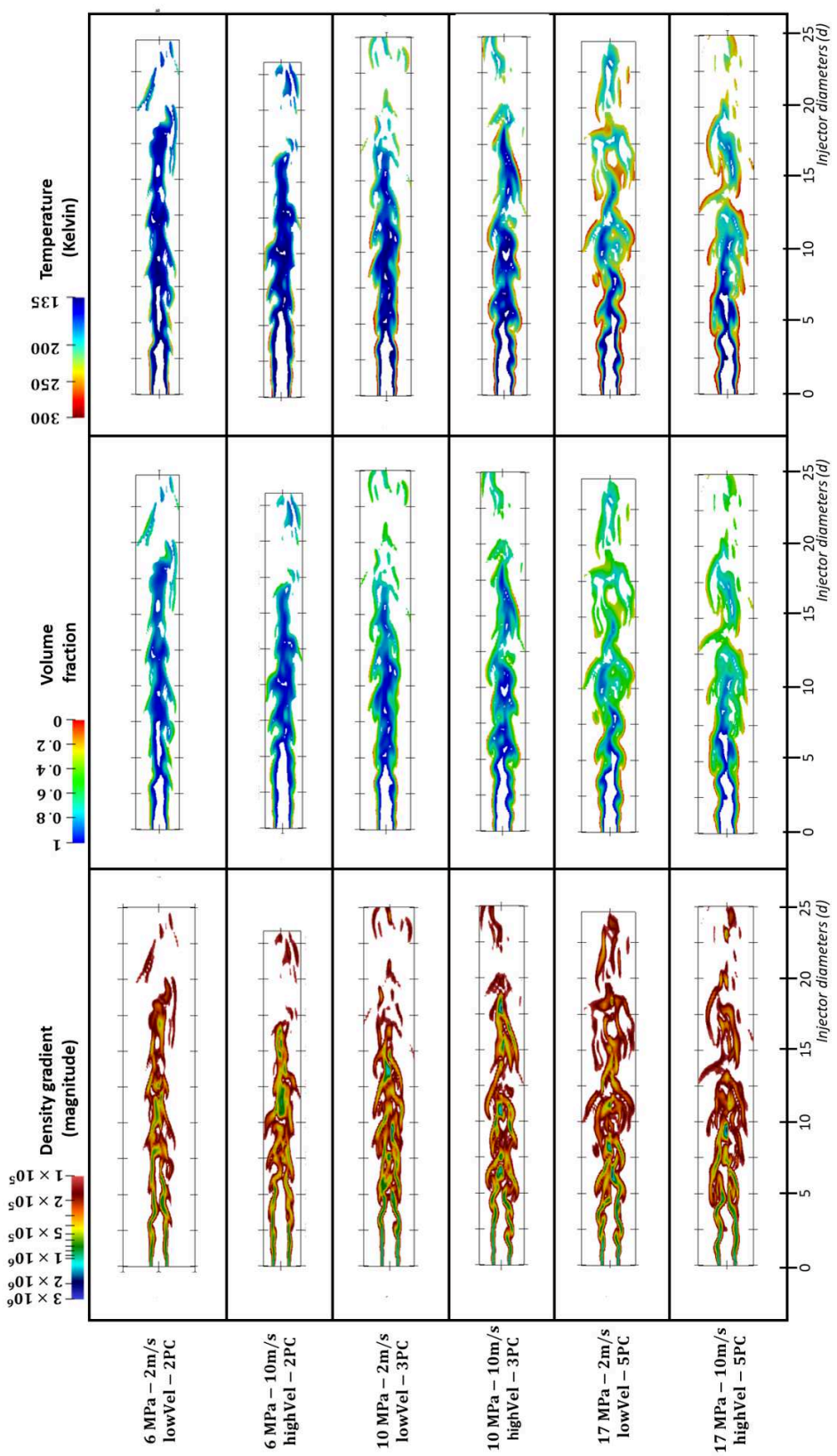

FIG. 16. Axial cross-section of density gradient tubes and the distribution of temperature and volume fraction across them 

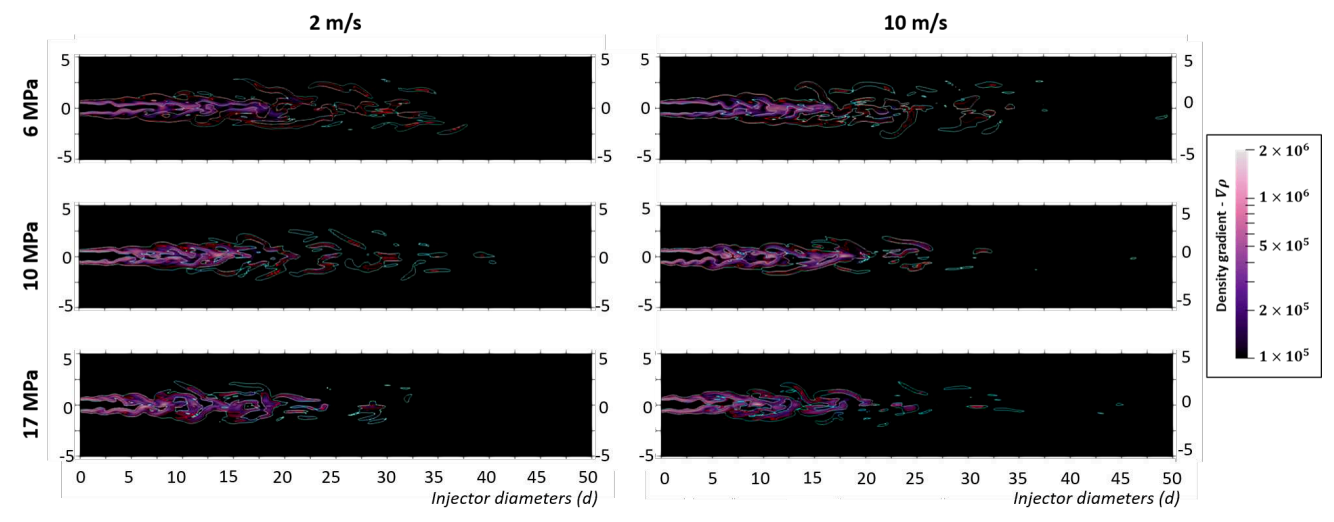

FIG. 17. Highest order density gradients colored by their strength. The boundaries of the highest order mass flux due to molecular diffusion (light blue) and thermo(Soret)-diffusion (red) are also included.

lent jets it is also the area where the flow is dominated by Kelvin-Helmholtz-type structures. In previous works on turbulent jets the exact location of the end of the potential core is assessed by various criteria depending on the properties of the jet. There can be different potential core regions for different properties. The criteria to determine the length of the potential core are velocity decay ${ }^{58}$, density decay ${ }^{58}$ and even species' concentration decay. The fundamental basis of the potential core is to identify the region where the injected fluid is still not mixing with the ambient fluid. In the cryogenic jets a similar region (up to $x / d=5$ ) is present where the density as well as the velocity are constant. These constant characteristics might not be enough to characterise the area as "potential core" since for supercritical cryogenic jets neither velocity decay nor density decay changes due to mixing alone. These properties are also influenced by thermodynamic transitions. This also means that traditional mechanical based breakup formulations $\left(f(R e)\right.$ or $f\left(U / U_{i n j}\right)$ or $\left.f\left(\rho_{i n j} / \rho_{\infty}\right)\right)$ can't estimate the potential core in supercritical cryogenic jets and a thermal driven transition mechanism needs to be accounted for, which is in line with Banuti's ${ }^{15}$ conclusions. Lack of sufficient number of experimental measurements especially at the injection location is a significant hurdle to formulate estimation methods for potential core in such supercritical cryogenic jets. Another difference in this near nozzle area is that the density gradients suppress the development of initial fluctuation. With increase of pressure in cryogenic jets the phase related, velocity related and density related potential cores become smaller (see Fig. 8 and 10).

Two of the existing criteria to estimate the potential core of fluid jets from the centreline velocity distribution are: The boundary where the velocity decays to $0.9-0.99$ of the injected velocity ${ }^{59,60}$, and the other one is where the asymptote (or extrapolation) of velocity decay intercepts the no decay $\left(U / U_{i n j}=1\right.$, see 61$)$. This is applicable to incompressible jets, but for compressible jets, the centerline density ra- tio (with respect to injection density) is a more appropriate criterion $^{62}$. For such compressible and incompressible jets, theoretical estimation methods for potential core length are functions of velocity ratio (or Reynolds number) and density ratio $\left[x_{p c}=f(R e)\right.$ or $\quad x_{p c}=f\left(U / U_{i n j}\right)$ and $\quad x_{p c}=$ $\left.f\left(\rho_{i n j} / \rho_{\infty}\right)\right]$ (see 15 and 58$)$. The empirical constants or coefficients in these functions are determined from appropriate experiments ${ }^{58}$. The potential core estimations for the simulation cases based on theory for both compressible and incompressible jets are given in Table VIII, and compared against the potential core obtained in our simulations. Both the theoretical methods for compressible and incompressible jets do not correspond with the simulation results which is rather expected given that these relations take into account advection controlled mixing alone. In supercritical cryogenic jets injected close to critical temperature, there is a greater influence of thermodynamic transition of the fluid and its properties from liquid/liquid-like to gas-like affecting the density and velocity decay. In addition molecular and Soret diffusion are also expected to influence the potential core.

The transitional area is the region between the near-field and the far field turbulent jet where vorticity has broken down into complex structures and the evolution of a cascade to smaller scales has begun. In this region it may be possible to observe more clearly the interaction between the vortex dynamics and the evolution of the turbulence into similarity. In Fig. 13 for the cryogenic jets we can see that after $x / d=5$ for all cases the vorticity breaks down and stops aligning with the density gradients.

In terms of the rms of the velocity, gas jets follow an identical (to cryogenic jets) trend demonstrating an initial suppression followed by a peak ${ }^{58,63}$ while for liquid jets there is a constant reduction of the rms with downstream distance due to the dissipation of turbulent kinetic energy ${ }^{64}$. In a gas jet into another gas the magnitude of the peak is influenced by the density difference between the injected and chamber gas. In our 
case the pick in terms of magnitude is rather unaffected by the pressure (which affects the density difference) although the location of the pick changes. Stronger density gradients result in more pronounced axial rms velocity peaks as the radial turbulence is transferred to axial direction. This can be verified with results from Satuet and Stepowski's experiments ${ }^{58,63}$ of gas jet injection into another gas, where large density gradients due to mixing between injected lighter gas (with respect to air) and surrounding air results in more prominent axial rms velocity peaks than radial rms velocity peaks, similar to our simulation results of supercritical jets in Fig. 11.

Fully turbulent self-similar regime is identified as the area where normalised flow properties collapse into a single curve. In incompressible gas jet calculations based 65 , self similarity can be defined when $U_{\text {inf }} / U \approx 1$ (where $U_{\text {inf }}$ is the velocity of the surrounding/co-flow gas). These jets can attain self similar characteristics at approximately $x / d>40$ (66). For liquid jets ( 64 and 67), experiments at atmospheric pressure conditions have shown the dependence of the self similar region on the Re numbers. The higher the Re numbers the quicker the transition to self-similar behaviour. For cryogenic jets we can define an equivalent to the classical definition of the self similar region, based on densities: $\rho_{\text {inf }} / \rho \approx 1$, following the concept of the effective diameter introduced in 68. Looking at Fig. 10 and Fig. 9 we can see that based on the density definition, a self similarity regions for thermophysical properties appears at $x / d>30$. Looking at velocities this region appears further downstream $(x / d>50)$. One difference with sub critical turbulent jets is that the mixing in the case of supercritical cryogenic jets is more enhanced by the increase of pressure which however leads to lower Re numbers. Isotropy in supercritical nitrogen jets is only reached at some distance beyond $50 d_{i n j}$ downstream due to the turbulence kinetic energy transfer (from radial to axial) by density gradients. The density gradients and the trend in turbulent fluctuations of supercritical $\mathrm{N}_{2}$ jets resemble more of subcritical gas jets mixing into another gas.

\section{CONCLUSION}

The evolution of cryogenic single fluid jets -initially at subcritical temperatures- which are injected into a supercritical environment has been investigated numerically in our work. For the first time we investigate together the thermophysical and dynamic behaviour of these jets and compare this behaviour with other gas and liquid jets. For the simulations we introduce a new numerical framework based on OpenFOAM which accounts for compressible, real fluid properties and additionally, in contrast to previous frameworks, diffusion due to both Soret and molecular effects are also included. The conclusions of our work can be summarised as following.

- A new phase characterisation framework based on association of phases with specific temperature ranges (rather than using a single line like the Widom line) has been suggested which allowed us to better identify self similarity effects between the various conditions.
- The thermophysical properties of the injected fluid influences the flow development more than the injected velocities. With increase in pressure the jets start to decay earlier and quicker. This also corresponds with increasingly steeper growth of fluctuations associated with increase in pressure.

- The non-dimensional centreline density measurements reveals that by keeping the injection temperature and chamber temperature fixed, the supercritical nitrogen jets follow identical thermally driven transition routes over wide range of pressures $\left(1.8 P_{c r}\right.$ to $\left.5 P_{c r}\right)$.

- We have identified a potential mechanism that as the density gradient tubes collapse, they lose their ability to suppress both axial and radial fluctuations. The remaining density gradient regions beyond the collapse slows the growth of radial fluctuations much more effectively (than axial fluctuations) due to the higher strength of these density gradient regions in the radial direction.

- The strong density gradients arising downstream of the injection suppresses radial fluctuations much more effectively, so even if the jet is injected at liquid state it will retain the non-isotropy for significantly large downstream distances unlike sub-critical liquid jets. With respect to the density gradient formation and turbulence development, a cryogenic fluid injected into the same fluid at supercritical conditions, behaves more similar to a gas jet into another gas with significant difference in their densities.

- Additionally, a conclusion of this analysis that is relevant to cooling applications is that at lower supercritical pressures the cryogenic jets penetrate deeper absorbing heat without immediately changing phase. On the other hand at high supercritical pressures these jets heat up faster resulting in gaseous jets quicker.

Coming back to the three initial questions presented in introduction, which we aimed to find answers in this research, the above conclusions infer that

1. In supercritical cryogenic jets, thermodynamics play a much more dominant role compared to fluid mechanics, especially at near critical conditions explored in this research. In addition, the mechanical properties such as density and viscosity which influence the fluid mechanics, depend on the fluid's thermodynamic state as well. In this research we have showed that the thermodynamic supercritical phase transition has a major influence on the jet evolution and characteristics.

2. While cryogenic jets easily attain high Re numbers at injection due to the associated high density and low viscosity, they do not behave like high Re jets in atmospheric conditions. Even while comparing cryogenic jets of Re with 5 times difference in this research, we cannot observe any difference in turbulence characteristics, as even the turbulent fluctuations (nondimensionalised) develop very similarly. 


\begin{tabular}{|c|c|c|c|c|c|c|c|}
\hline \multirow{2}{*}{$\begin{array}{l}\text { Simulation } \\
\text { case }\end{array}$} & \multirow{2}{*}{$\begin{array}{c}\text { Pressure } \\
(\mathrm{MPa})\end{array}$} & \multirow[t]{2}{*}{$\rho_{0} / \rho_{\infty}$} & \multirow{2}{*}{$\begin{array}{c}\text { Reynolds no } \\
\quad \operatorname{Re}\end{array}$} & \multicolumn{2}{|c|}{ Potential core length $-x_{p} / d_{d i n j}$} & \multicolumn{2}{|c|}{ Potential core length $(\operatorname{Sim})-x_{p} / d_{d i n j}$} \\
\hline & & & & $=5.4 \sqrt{\rho_{\text {inj }} / \rho_{\infty}}$ & $=1+1.7 \times 10^{-4} \operatorname{Re}$ & at $\left(U / U_{i n j}\right)=0.9$ & at $\rho^{* 10}=0.9$ \\
\hline lowVel-2Pc & $6\left(1.8 P_{c r}\right)$ & 6.32 & $6.75 \times 10^{4}$ & 13.6 & 12.5 & 8.55 & 6.75 \\
\hline highVel-2Pc & $6\left(1.8 P_{c r}\right)$ & 6.32 & $33.63 \times 10^{4}$ & 13.6 & 58.2 & 9.25 & 7.3 \\
\hline lowVel-3Pc & $10\left(3 P_{c r}\right)$ & 4.77 & $5.80 \times 10^{4}$ & 11.8 & 10.9 & 7.5 & 6.45 \\
\hline highVel-3Pc & $10\left(3 P_{c r}\right)$ & 4.77 & $29.02 \times 10^{4}$ & 11.8 & 50.3 & 7.75 & 7.25 \\
\hline lowVel-5Pc & $17\left(5 P_{c r}\right)$ & 3.27 & $5.08 \times 10^{4}$ & 9.8 & 9.6 & 6.55 & 6.75 \\
\hline highVel-5Pc & $17\left(5 P_{c r}\right)$ & 3.27 & $25.40 \times 10^{4}$ & 9.8 & 44.2 & 5.25 & 5 \\
\hline
\end{tabular}

TABLE VIII. Comparison of potential core estimation from theoretical models for usual fluid jets ${ }^{58}$, with results of our simulation of supercritical $\mathrm{N}_{2}$ jet

3. The formation of density gradients and the trend in development of turbulent fluctuations point out that these transitioning supercritical cryogenic jets behave more like subcritical gas jet injected into another gas, although the density gradients in cryogenic jets are due to the combined effect of mixing (between injected cryogenic fluid and chamber fluid) as well as thermodynamic transition of the fluid (towards supercritical gaslike/ideal gas-like). In addition the absence of surface tension, which is one of the main properties influencing the subcritical liquid jet breakup, also makes cryogenic jets behave much more like gas jets.

We remind that is this work we focus on single-specie system injected and chamber specie - $\mathrm{N}_{2}$ ) to avoid complexities in the result interpretation relevant to multi-species effects such as definition of surface tension and the critical point of the mixture at the interface between the injected and the chamber fluid. Such a system in reality can be indicative also of some binary mixtures exhibiting miscible type-I mixing behaviour (such as $\mathrm{Ar} / \mathrm{Kr}$, where both substances are of similar chemical types and/or their critical properties are comparable in magnitude.) as demonstrated from the work of Raju et al. ${ }^{25}$. Future work will include extending our analysis to binary mixtures.

Since the experimental data for such supercritical cryogenic jets is very limited, an high pressure chamber with optical access is also being assembled at University of Brighton. This would enable us to perform in-depth in-house experiments on supercritical jets in the near future, and provide us with necessary experimental data for validation.

\section{DATA AVAILABILITY}

The data that supports the findings of this study are available within the article

\section{ACKNOWLEDGMENTS}

The authors would like to acknowledge funding by the UK Engineering and Physical Science Research Council support through the grant EP/S001824/1
Appendix A: 'coolFoam' solver characteristics

The general characteristics of the 'coolFoam' solver (based on OpenFOAM framework) including the numerical schemes and thermophysical models are presented in Table IX.

\begin{tabular}{|c|c|}
\hline \multicolumn{2}{|c|}{ General characteristics } \\
\hline coolFoam & $\begin{array}{l}\text { Eulerian VoF for } 2 \text { compressible } \\
\text { miscible/immiscible fluids }\end{array}$ \\
\hline Algorithm & PIMPLE (Pressure based segregated) \\
\hline \multicolumn{2}{|c|}{ Conservation equations } \\
\hline Phase transport & In terms of volume fraction $(\alpha)$ \\
\hline Momentum & $\begin{array}{l}\text { Includes surface tension and } \\
\text { gravity effects }\end{array}$ \\
\hline Energy & Conservation of Internal Energy \\
\hline \multicolumn{2}{|c|}{ Discretisation schemes } \\
\hline Time & First order Euler \\
\hline Convection & Second order Gauss limited-linear \\
\hline Diffusion & Second order Gauss limited-linear \\
\hline \multicolumn{2}{|c|}{ Thermophysical models } \\
\hline Density & Polynomial fit of NIST data \\
\hline Thermodynamic properties & Polynomial fit of NIST data \\
\hline Transport properties & Polynomial fit of NIST data \\
\hline Mixture properties & Volume fraction weighted \\
\hline Diffusion & mass (molecular and Soret) \\
\hline
\end{tabular}

TABLE IX. Solver and numerical setup characteristics

\section{Appendix B: Grid dependence study}

The grid dependency study was performed utilising 3 meshes of varying refinement and with the same domain and boundary conditions. Brief details of these 3 meshes are presented in Table X. The cell to cell expansion ratio for all the three meshes are kept identical, so that similar grading exists across all the meshes, but with varying number of cells.

The time averaged axial centreline density distribution simulation results of the case 'lowVel-2Pc' with the 3 meshes of varying refinement are presented in Fig. 18 along with the experimental data (refer Section III B for further details about the case and experiment). It can be seen from the Fig. 18 that the results of the simulation mesh improve significantly compared with the coarse mesh. Whereas there is only minor 

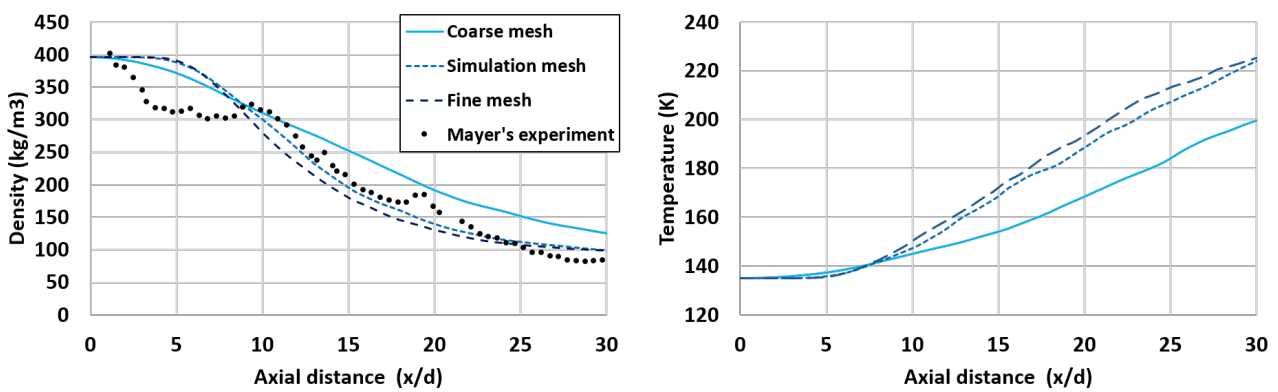

FIG. 18. Axial centreline distribution of density (left) and temperature (right) for the case 'lowVel-2Pc' using 3 meshes of varying refinement. Experimental density measurements (refer Section III B) which corresponds to our case 'lowVel-2Pc' is also plotted.

\begin{tabular}{cccccc}
\hline \hline Mesh & No of cells & $\begin{array}{c}\text { Min cell vol } \\
\left(\mathrm{m}^{3}\right)\end{array}$ & $\begin{array}{c}\text { Max cell vol } \\
\left(\mathrm{m}^{3}\right)\end{array}$ & $\begin{array}{c}\sqrt[3]{(\text { Mincellvol })} \\
(\mathrm{m})\end{array}$ & $\begin{array}{c}\sqrt[3]{\text { Maxcell vol }} \\
(\mathrm{m})\end{array}$ \\
\hline Coarse mesh & & $1.102 \times 10^{-11}$ & $8.993 \times 10^{-7}$ & $2.225 \times 10^{-4}$ & $9.652 \times 10^{-3}$ \\
Simulation mesh & 256000 & $1.953 \times 10^{-12}$ & $2.606 \times 10^{-7}$ & $1.250 \times 10^{-4}$ & $6.388 \times 10^{-3}$ \\
Fine mesh & 976000 & $4.331 \times 10^{-13}$ & $1.477 \times 10^{-7}$ & $7.566 \times 10^{-5}$ & $5.286 \times 10^{-3}$ \\
\hline \hline
\end{tabular}

TABLE X. Grid details of meshes used in grid dependency study.

differences downstream (from 7.5d) comparing the results of simulation mesh and fine mesh, even though the fine mesh has 2 times more number of cells than the simulation mesh and the cell size is almost halved in the grid domain near the injection. Thus 'Simulation mesh' provides sufficient accuracy while considering the computational cost and hence is utilised for this study.

${ }^{1}$ R. Morgan, C. Lenartowicz, K. Vogiatzaki, S. Harvey, D. Kennaird, N. Owen, R. Pickett, and A. Atkins, "The ultra low emissions potential of the recuperated split cycle combustion system," in SAE Technical Paper (SAE International, 2019) pp. 2019-24-0189.

2"Spacex," Available online: https://www.spacex.com/vehicles/starship/ (accessed on 22 November 2020).

3"Be-4," Available online: https://www.blueorigin.com/engines/be-4 (accessed on 22 November 2020).

${ }^{4}$ B. Rubinsky, "Cryosurgery," Annual Review of Biomedical Engineering 2 157-187 (2000)

${ }^{5}$ L. Gao, L. Yang, S. Qian, Z. Tang, W. Qin, F., H. Q., and J. P., Yuan, "Cryosurgery would be an effective option for clinically localized prostate cancer: A meta-analysis and systematic review," Scientific Reports 6 27490 (2016)

${ }^{6}$ M. Jaya Vignesh, G. Tretola, R. Morgan, G. d. Sercey, A. Atkins, an

K. Vogiatzaki, "Understanding sub and supercritical cryogenic fluid dy namics in conditions relevant to novel ultra low emission engines," Energies 13, 3038 (2020).

${ }^{7}$ H. Liu and M. Altan, "Science and Engineering of Droplets: Fundamental and Applications," Applied Mechanics Reviews 55, B16-B17 (2002).

${ }^{8}$ W. Mayer, A. Schik, C. Schweitzer, and M. Schaeffler, "Injection and mixing processes in high pressure lox/gh2 rocket combustors," in 32nd Joint Propulsion Conference and Exhibit.

${ }^{9}$ W. O. H. Mayer, A. H. A. Schik, B Vielle, C. Chauveau, I. G-ograve, kalp, D. G. Talley, and R. D. Woodward, "Atomization and breakup of cryogenic propellants under high-pressure subcritical and supercritical conditions," Journal of Propulsion and Power 14, 835-842 (1998).

${ }^{0}$ W. Mayer, J. Telaar, R. Branam, and G. Schneider, "Characterization of cryogenic injection at supercritical pressure," in 37th Joint Propulsion Conference and Exhibit (2001).

${ }^{11}$ B. Chehroudi, D. Talley, and E. Coy, "Fractal geometry and growth rate changes of cryogenic jets near the critical point," in 35th Joint Propulsion Conference and Exhibit.

${ }^{12} \mathrm{M}$. Oschwald and A. Schik, "Supercritical nitrogen free jet investigated by spontaneous raman scattering," Experiments in Fluids 27, 497-506 (1999). ${ }^{13}$ M. Oschwald, A. Schik, M. Klar, and W. Mayer, "Investigation of coaxial $\ln 2 / g h 2$-injection at supercritical pressure by spontaneous raman scattering," in 35th Joint Propulsion Conference and Exhibit.

${ }^{14}$ J. A. Newman and T. A. Brzustowski, "Behavior of a liquid jet near the thermodynamic critical region," AIAA Journal 9, 1595-1602 (1971).

${ }^{15}$ D. T. Banuti and K. Hannemann, "The absence of a dense potential core in supercritical injection: A thermal break-up mechanism," Physics of Fluids 28, 035103 (2016)

${ }^{16}$ D. Banuti, "The latent heat of supercritical fluids," Periodica Polytechnica Chemical Engineering 63 (2019), 10.3311/PPch.12871.

${ }^{17} \mathrm{D}$. Banuti, M. Raju, and M. Ihme, "Between supercritical liquids and gases - reconciling dynamic and thermodynamic state transitions," The Journal of Supercritical Fluids 165, 104895 (2020).

${ }^{18}$ G. Lamanna, I. Stotz, B. Weigand, and J. Steelant, "Supercritical Fluid Injection: An Experimental Study," in 7th European Symposium on Aerothermodynamics, ESA Special Publication, Vol. 692, edited by L. Ouwehand (2011) p. 30 .

${ }^{19}$ G. Lamanna, H. Kamoun, B. Weigand, and J. Steelant, "Towards a unified treatment of fully flashing sprays," International Journal of Multiphase Flow 58, 168-184 (2014)

${ }^{20}$ G. Lamanna, H. Kamoun, B. Weigand, C. Manfletti, A. Rees, J. Sender, M. Oschwald, and J. Steelant, "Flashing behavior of rocket engine propellants," Atomization and Sprays 25, 837-856 (2015).

${ }^{21}$ G. Lamanna, "Cryogenic flashing jets: A review (invited paper)," in 52nd AIAA/SAE/ASEE Joint Propulsion Conference.

${ }^{22}$ J. W. Gärtner, A. Kronenburg, A. Rees, J. Sender, M. Oschwald, and G. Lamanna, "Numerical and experimental analysis of flashing cryogenic nitrogen," International Journal of Multiphase Flow 130, 103360 (2020).

${ }^{23}$ G. Lamanna, C. Steinhausen, B. Weigand, A. Preusche, B. Bork, A. Dreizler, R. Stierle, and J. Groß, "On the importance of non-equilibrium models 
for describing the coupling of heat and mass transfer at high pressure," International Communications in Heat and Mass Transfer 98, 49 - 58 (2018). ${ }^{24}$ A. Preusche, A. Dreizler, C. Steinhausen, G. Lamanna, and R. Stierle, "Non-invasive, spatially averaged temperature measurements of falling acetone droplets in nitrogen atmosphere at elevated pressures and temperatone droplets in nitrogen atmosphere at elevated pressures and
tures," The Journal of Supercritical Fluids 166, 105025 (2020).

${ }^{25}$ M. Raju, D. Banuti, P. Ma, and M. Ihme, "Widom lines in binary mixtures of supercritical fluids," Scientific Reports 7 (2017), 10.1038/s41598-01703334-3

${ }^{26}$ G. Simeoni, T. Bryk, F. Gorelli, M. Krisch, G. Ruocco, M. Santoro, and T. Scopigno, "The widom line as crossover between liquid-like and gas-like behaviour in supercritical fluids," Nature Physics 6, 503-507 (2010).

${ }^{27}$ F. Gorelli, M. Santoro, T. Scopigno, M. Krisch, and G. Ruocco, "Liquidlike behavior of supercritical fluids," Phys. Rev. Lett. 97, 245702 (2006).

${ }^{28}$ F. Sciortino, P. Poole, U. Essmann, and H. Stanley, "Line of compressibility maxima in the phase diagram of supercooled water," Phys. Rev. E 55 (1997), 10.1103/PhysRevE.55.727.

${ }^{29} \mathrm{E}$. Lemmon, "Thermophysical properties of fluid systems, nist chemistry webbook, nist standard reference database number 69," http://webbook. nist. gov. (2005).

${ }^{30}$ L. Xu, P. Kumar, S. V. Buldyrev, S.-H. Chen, P. H. Poole, F. Sciortino, an H. E. Stanley, "Relation between the widom line and the dynamic crossove in systems with a liquid-liquid phase transition," Proceedings of the National Academy of Sciences 102, 16558-16562 (2005).

${ }^{31}$ M. E. Fisher and B. Widom, "Decay of correlations in linear systems," The Journal of Chemical Physics 50, 3756-3772 (1969).

${ }^{32} \mathrm{~K}$. Nishikawa and I. Tanaka, "Correlation lengths and density fluctuation in supercritical states of carbon dioxide," Chemical Physics Letters 244 $149-152(1995)$

${ }^{33}$ V. V. Brazhkin, Y. D. Fomin, A. G. Lyapin, V. N. Ryzhov, and K. Trachenko, "Two liquid states of matter: A dynamic line on a phase diagram," Phys. Rev. E 85, 031203 (2012)

${ }^{34} \mathrm{D}$. T. Banuti, "A thermodynamic look at injection in aerospace propulsion systems," in AIAA Scitech 2020 Forum.

${ }^{35} \mathrm{C}$. Li, C. Crua, and K. Vogiatzaki, "Effect of the scale resolution on the two phase coupling characteristics of high speed evaporating sprays using les / eulerian-lagrangian methodologies," International Journal of Multiphase Flow 120, 103060 (2019).

${ }^{36} \mathrm{P}$. C. Ma, H. Wu, D. T. Banuti, and M. Ihme, "On the numerical behavio of diffuse-interface methods for transcritical real-fluids simulations," International Journal of Multiphase Flow 113, 231-249 (2019).

${ }^{37}$ H. G. Weller, G. Tabor, H. Jasak, and C. Fureby, "A tensorial approach to computational continuum mechanics using object-oriented techniques," Computers in Physics 12, 620-631 (1998).

${ }^{38}$ S. Navarro-Martinez, G. Tretola, M. Reza Yosri, K. Vogiatzaki, and R. Gordon, "An investigation on the impact of small-scale models in gasoline direct injection sprays (ecn spray g)," International Journal of Engine Research 21, 217-225 (2019).

${ }^{39}$ W. Mayer, J. Telaar, R. Branam, G. Schneider, and J. Hussong, "Raman measurements of cryogenic injection at supercritical pressure," Heat and Mass Transfer 39, 709-719 (2003).

${ }^{40} \mathrm{~N}$. Zong, H. Meng, S.-Y. Hsieh, and V. Yang, "A numerical study of cryogenic fluid injection and mixing under supercritical conditions," Physics of Fluids 16, 4248-4261 (2004)

${ }^{41}$ T. Kim, Y. Kim, and S.-K. Kim, "Numerical study of cryogenic liquid nitrogen jets at supercritical pressures," The Journal of Supercritical Fluids 56, 152-163 (2011)

${ }^{42}$ T. S. Park, "Les and rans simulations of cryogenic liquid nitrogen jets," The Journal of Supercritical Fluids 72, 232-247 (2012).

${ }^{43}$ X. Petit, G. Ribert, G. Lartigue, and P. Domingo, "Large-eddy simulation of supercritical fluid injection," The Journal of Supercritical Fluids 84, 61 73 (2013)

${ }^{44}$ W. Wei, M. Xie, and M. Jia, "Large eddy simulation of fluid injection under transcritical and supercritical conditions," Numerical Heat Transfer, Part A Applications 70, 870-886 (2016)

${ }^{45}$ H. Müller, C. A. Niedermeier, J. Matheis, M. Pfitzner, and S. Hickel,
"Large-eddy simulation of nitrogen injection at trans-and supercritical conditions," Physics of fluids 28, 015102 (2016).

${ }^{46}$ D.-Y. Peng and D. B. Robinson, "A new two-constant equation of state," Industrial \& Engineering Chemistry Fundamentals 15, 59-64 (1976).

${ }^{47} \mathrm{G}$. Soave, "Equilibrium constants from a modified Redlich-Kwong equation of state," Chemical Engineering Science 27, 1197-1203 (1972).

${ }^{48}$ T. H. Chung, M. Ajlan, L. L. Lee, and K. E. Starling, "Generalized multiparameter correlation for nonpolar and polar fluid transport properties," Industrial \& engineering chemistry research 27, 671-679 (1988).

${ }^{49}$ V. Gerber, S. Baab, F. J. Förster, H. Mandler, B. Weigand, and G. Lamanna, "Fluid injection with supercritical reservoir conditions: overview on morphology and mixing." The Journal of Supercritical Fluids , 105097 (2020).

${ }^{50}$ F. Föll, V. Gerber, C.-D. Munz, B. Weigand, and G. Lamanna, "On the consideration of diffusive fluxes within high-pressure injections," in Future Space-Transport-System Components under High Thermal and Mechanical Loads: Results from the DFG Collaborative Research Center TRR40, edited by N. A. Adams, W. Schröder, R. Radespiel, O. J. Haidn, T. Sattelmayer, C. Stemmer, and B. Weigand (Springer International Publishing, Cham, 2021) pp. 195-208.

${ }^{51}$ M. Jaya Vignesh, S. Harvey, A. Atkins, P. Atkins, G. De Sercey, M. Heikal, R. Morgan, and K. Vogiatzaki, "Use of cryogenic fluids for zero toxic emission hybrid engines," in Internal Combustion Engines and Powertrain Systems for Future Transport 2019: Proceedings of the International Conference on Internal Combustion Engines and Powertrain Systems for Future Transport,(ICEPSFT 2019), December 11-12, 2019, Birmingham, UK (CRC Press, 2020) p. 117.

${ }^{52}$ E. N. Fuller, P. D. Schettler, and J. C. Giddings, "New method for prediction of binary gas-phase diffusion coefficients," Industrial \& Engineering Chemistry 58, 18-27 (1966)

${ }^{53}$ J. K. Platten, "The Soret Effect: A Review of Recent Experimental Results," Journal of Applied Mechanics 73, 5-15 (2005).

${ }^{54}$ S. Takahashi, "Preparation of a generalized chart for the diffusion coefficients of gases at high pressures," Journal of Chemical Engineering of Japan 7, 417-420 (1975).

${ }^{55} \mathrm{M}$. Klein, A. Sadiki, and J. Janicka, "A digital filter based generation of inflow data for spatially developing direct numerical or large eddy simulations," Journal of computational Physics 186, 652-665 (2003).

${ }^{56} \mathrm{~L}$. Li, M. Xie, W. Wei, M. Jia, and H. Liu, "Numerical investigation on cryogenic liquid jet under transcritical and supercritical conditions," Cryogenics 89, 16 - 28 (2018)

${ }^{57}$ M. Oschwald, J. J. Smith, R. Branam, J. Hussong, A Schik, B. Chehroudi, and D. Talley, "Injection of fluids into supercritical environments," Combustion Science and Technology 178, 49-100 (2006).

${ }^{58}$ J. C. Sautet and D. Stepowski, "Dynamic behavior of variable-density, turbulent jets in their near development fields," Physics of Fluids 7, 2796-2806 (1995)

${ }^{59}$ R. C. Deo, J. Mi, and G. J. Nathan, "The influence of reynolds number on a plane jet," Physics of Fluids 20, 075108 (2008).

${ }^{60}$ T. L. Labus and E. P. Symons, "Experimental investigation of an axisymmetric free jet with an initially uniform velocity profile," (1972).

${ }^{61}$ K. B. M. Q. Zaman, "Asymptotic spreading rate of initially compressible jets-experiment and analysis," Physics of Fluids 10, 2652-2660 (1998).

${ }^{62} \mathrm{P}$. O. WITZE, "Centerline velocity decay of compressible free jets," AIAA Journal 12, 417-418 (1974).

${ }^{63}$ J. Sautet and D. Stepowski, "Single-shot laser mie scattering measurements of the scalar profiles in the near field of turbulent jets with variable densities," Experiments in fluids 16, 353-367 (1994).

${ }^{64} \mathrm{~A}$. Mansour and N. Chigier, "Turbulence characteristics in cylindrical liquid jets," Physics of Fluids 6, 3380-3391 (1994).

${ }^{65}$ Hinze, O., "Turbulence," in 2nd edition (McGraw-Hill, New York, 1975).

${ }^{66} \mathrm{~J}$. A. Schetz, "Injection and mixing in turbulent flow," Progress in Astronautics and Aeronautics 68 (1980).

${ }^{67} \mathrm{~L}$. D. Weisgraber, T., "Turbulent structure during transition to self-similarity in a round jet," Experiments in Fluids 24, 210-224 (1998).

${ }^{68} \mathrm{M}$. Thring and M. Newby, "Combustion length of enclosed turbulent jet flames," Symposium (International) on Combustion 4, 789 - 796 (1953), fourth Symposium (International) on Combustion. 


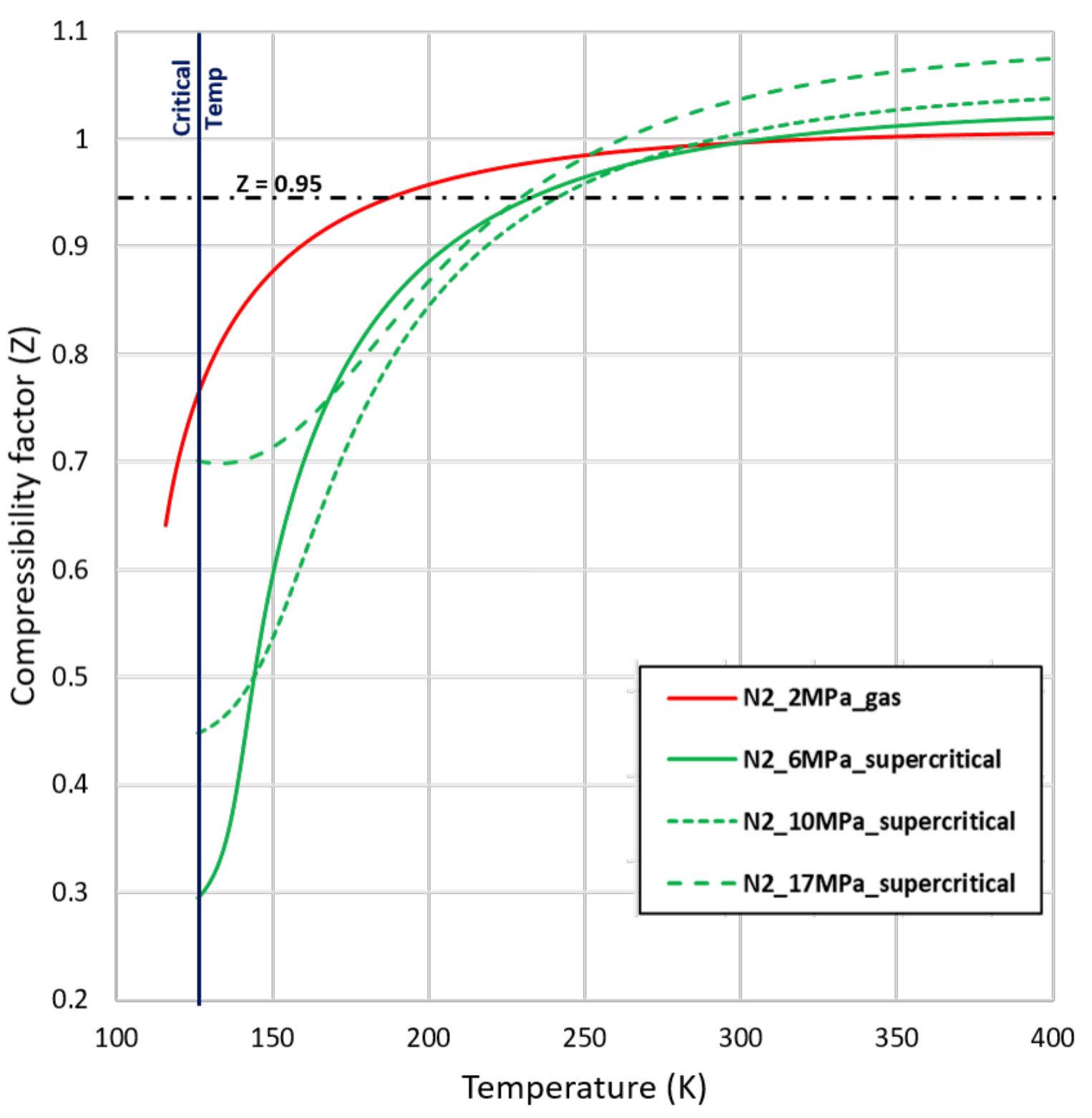



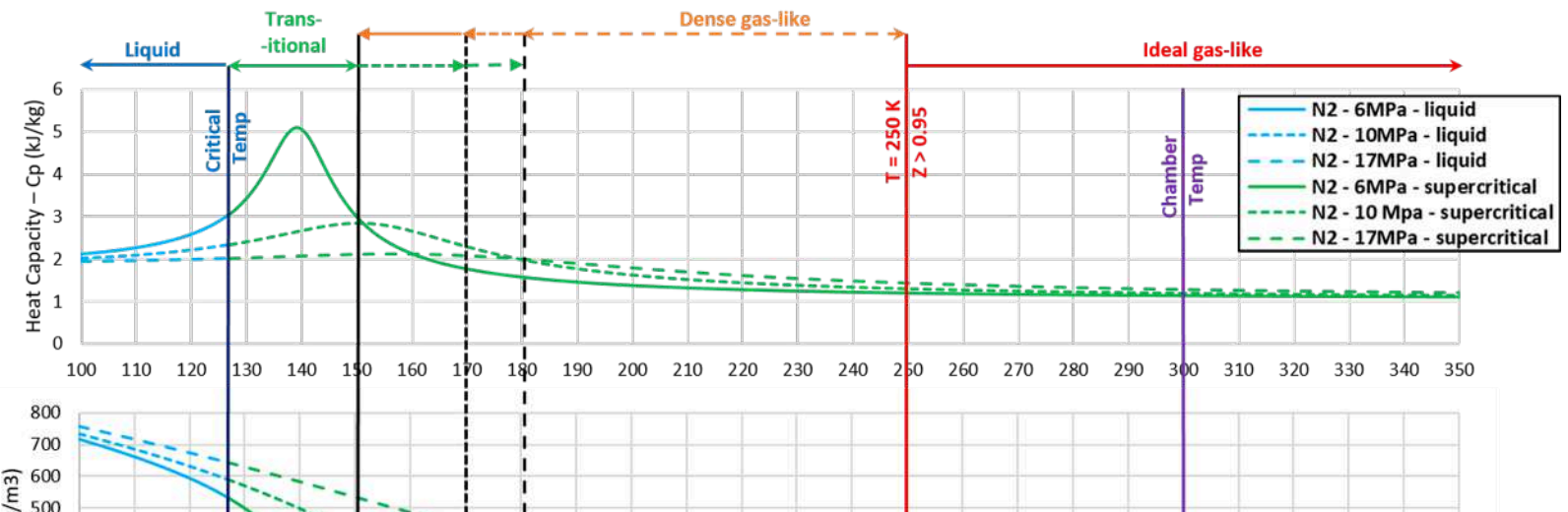

旁 400

ปั๊ 200

100

.
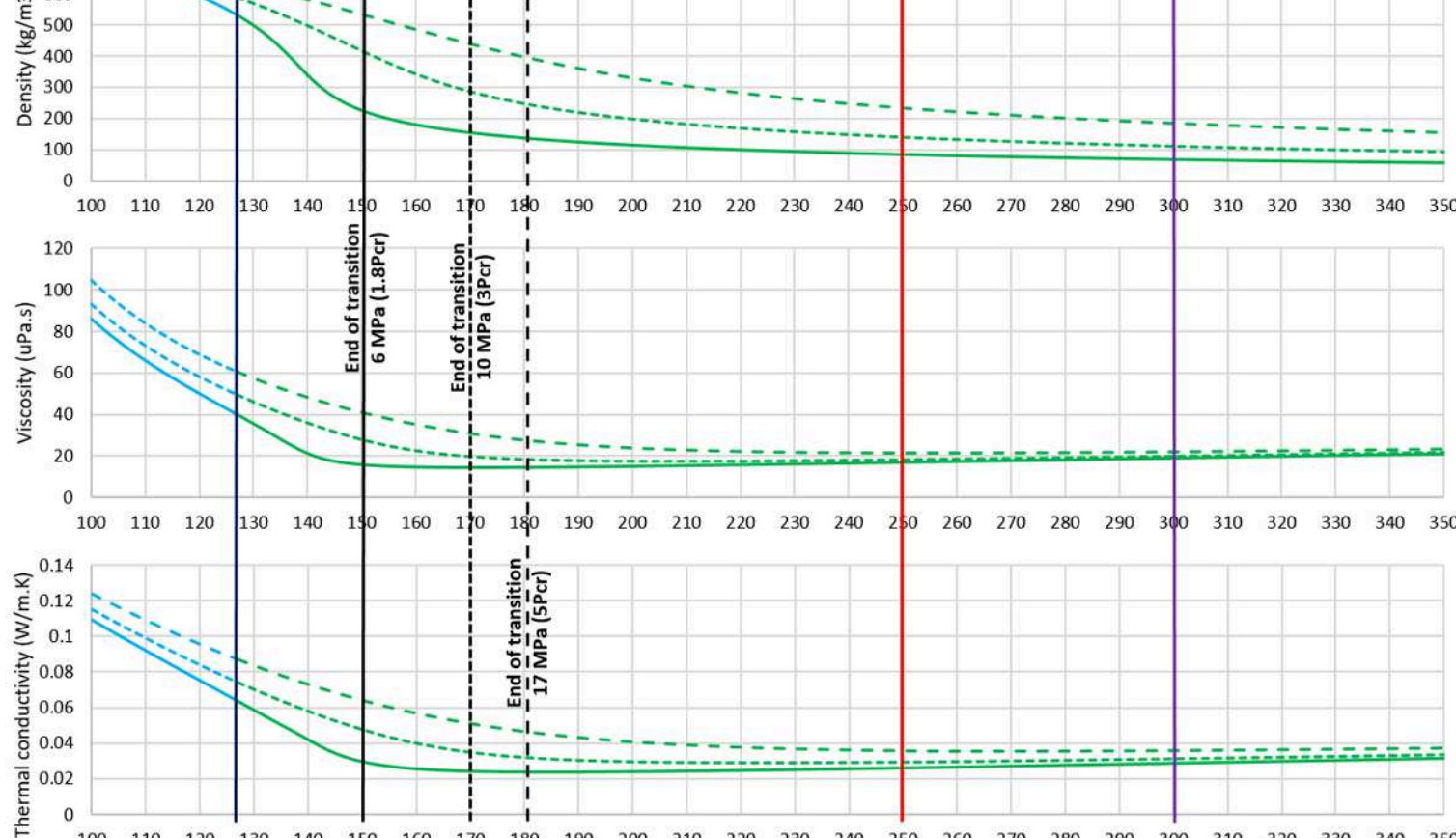
Temperature (K) 

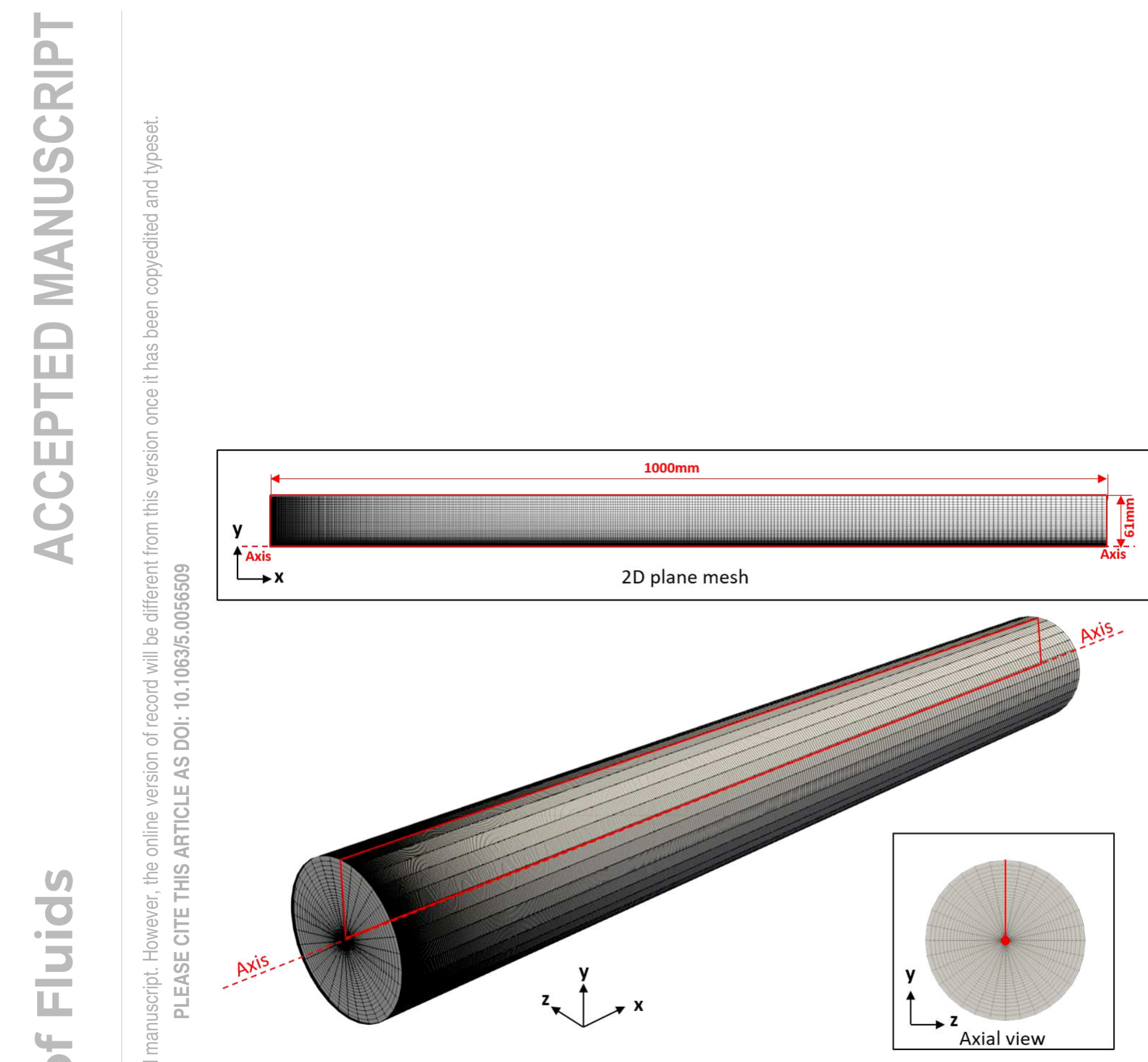

0
4
0
0
0

钫毫 

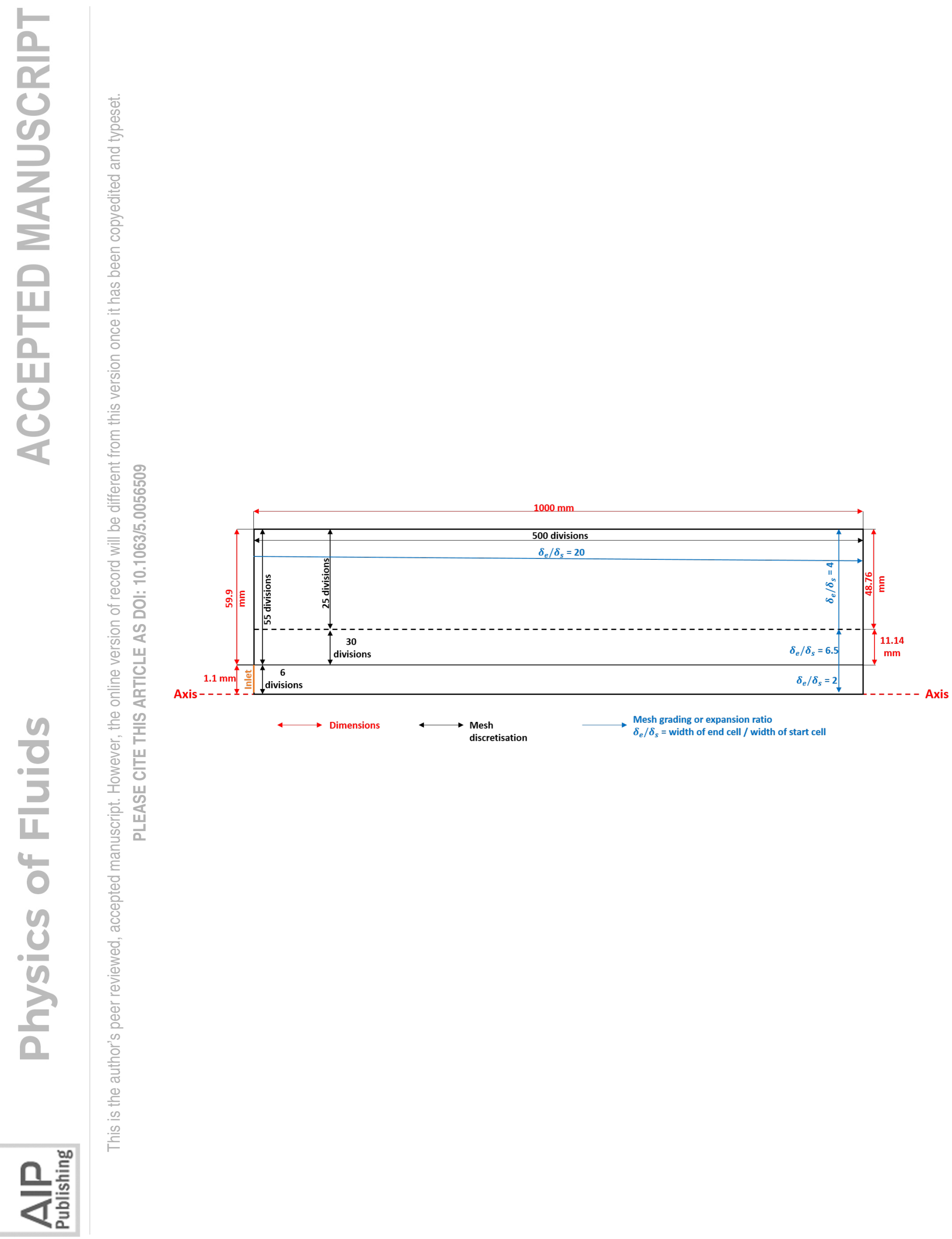

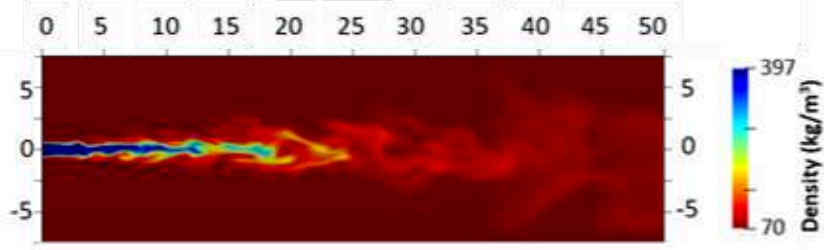

$\begin{array}{lllllllllll}0 & 5 & 10 & 15 & 20 & 25 & 30 & 35 & 40 & 45 & 50\end{array}$
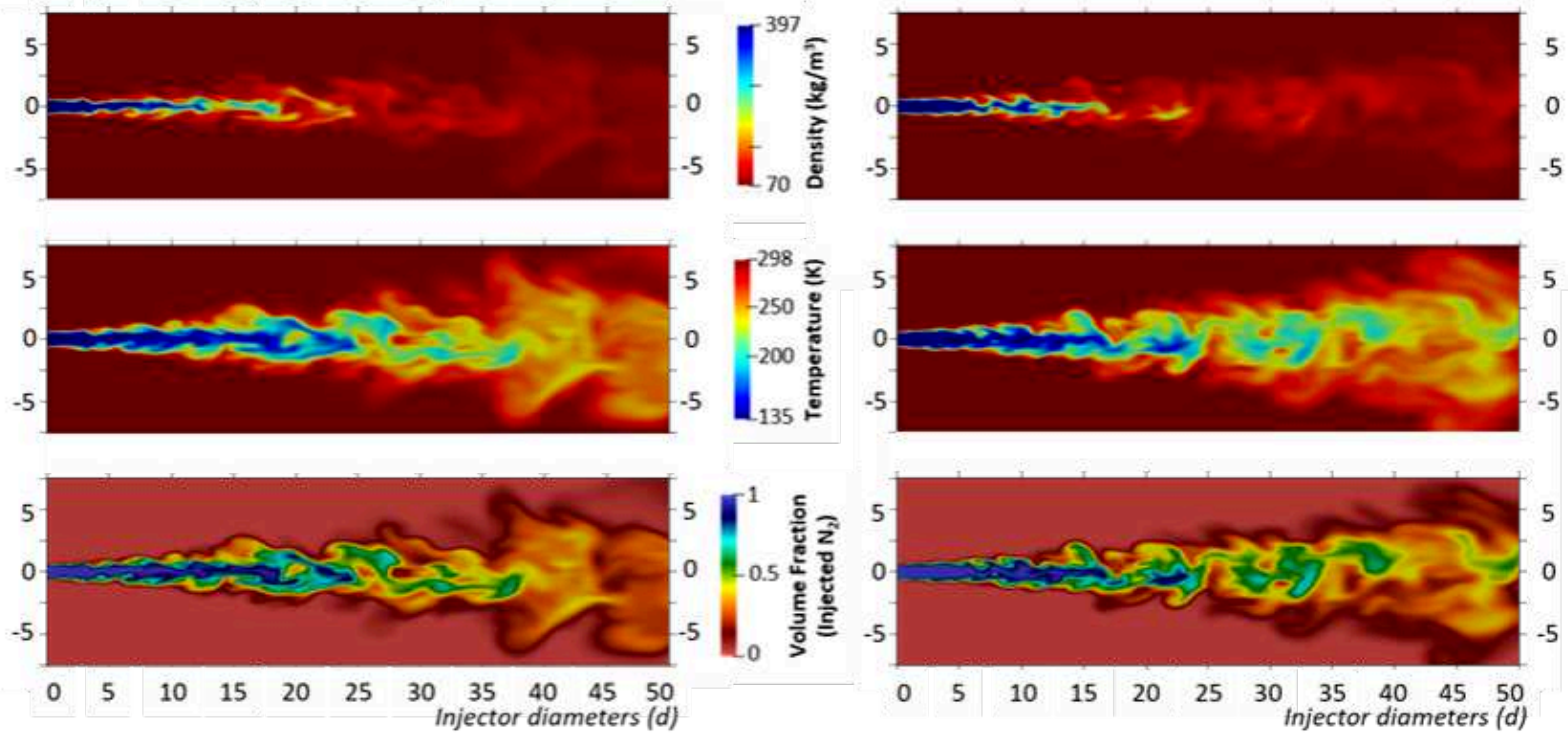


\section{$2 \mathrm{~m} / \mathrm{s}$}

$10 \mathrm{~m} / \mathrm{s}$
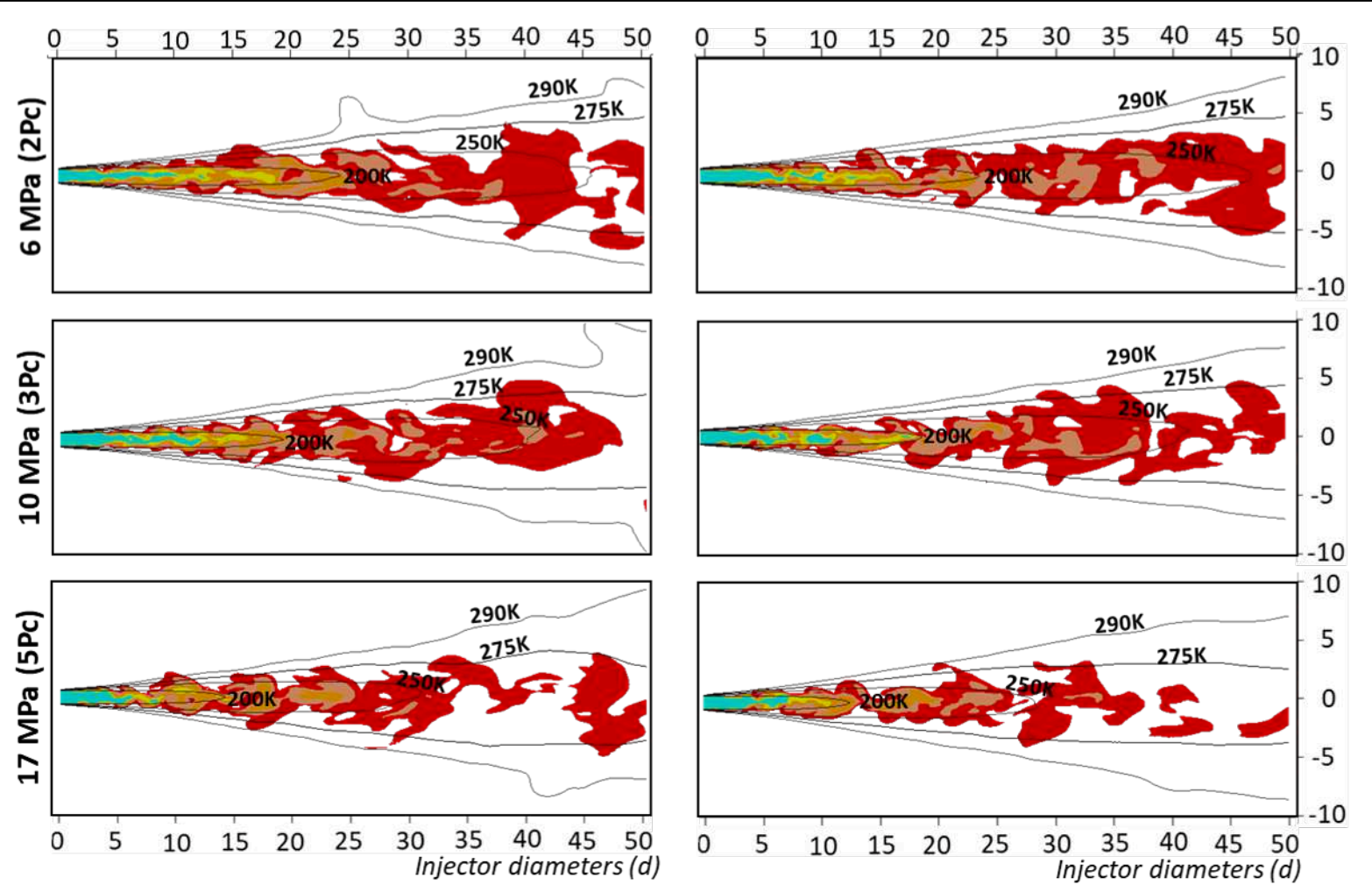

$\Delta H_{\text {available }}=\square 50-100 \mathrm{~kJ} / \mathrm{kg} \quad \square 100-150 \mathrm{~kJ} / \mathrm{kg} \quad \square 150-200 \mathrm{~kJ} / \mathrm{kg} \quad \square 200-250 \mathrm{~kJ} / \mathrm{kg} \quad \square 250-300 \mathrm{~kJ} / \mathrm{kg}$ 

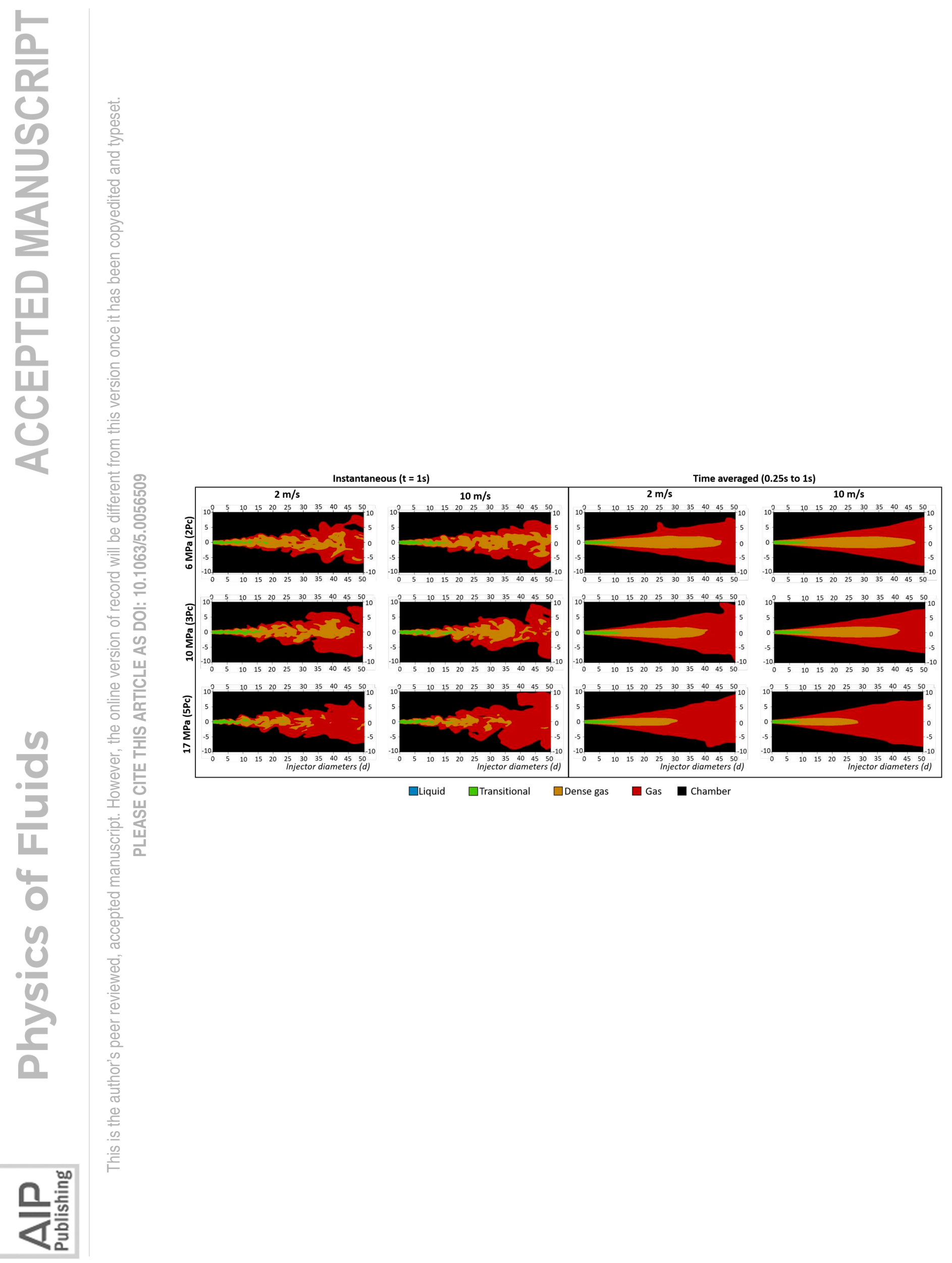

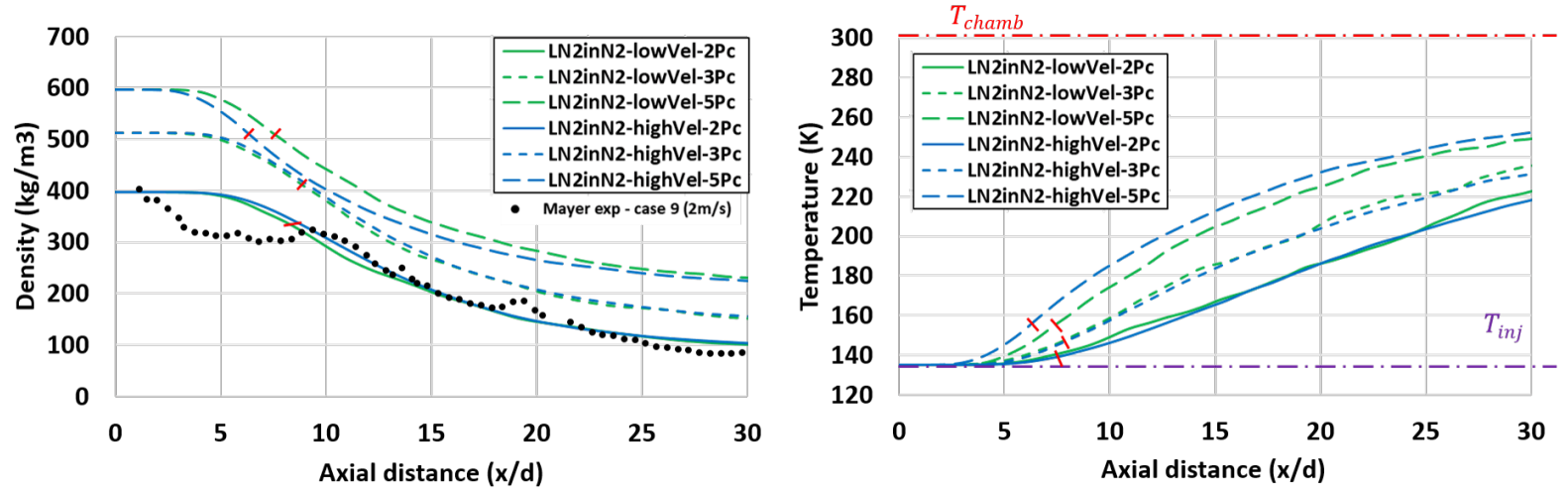

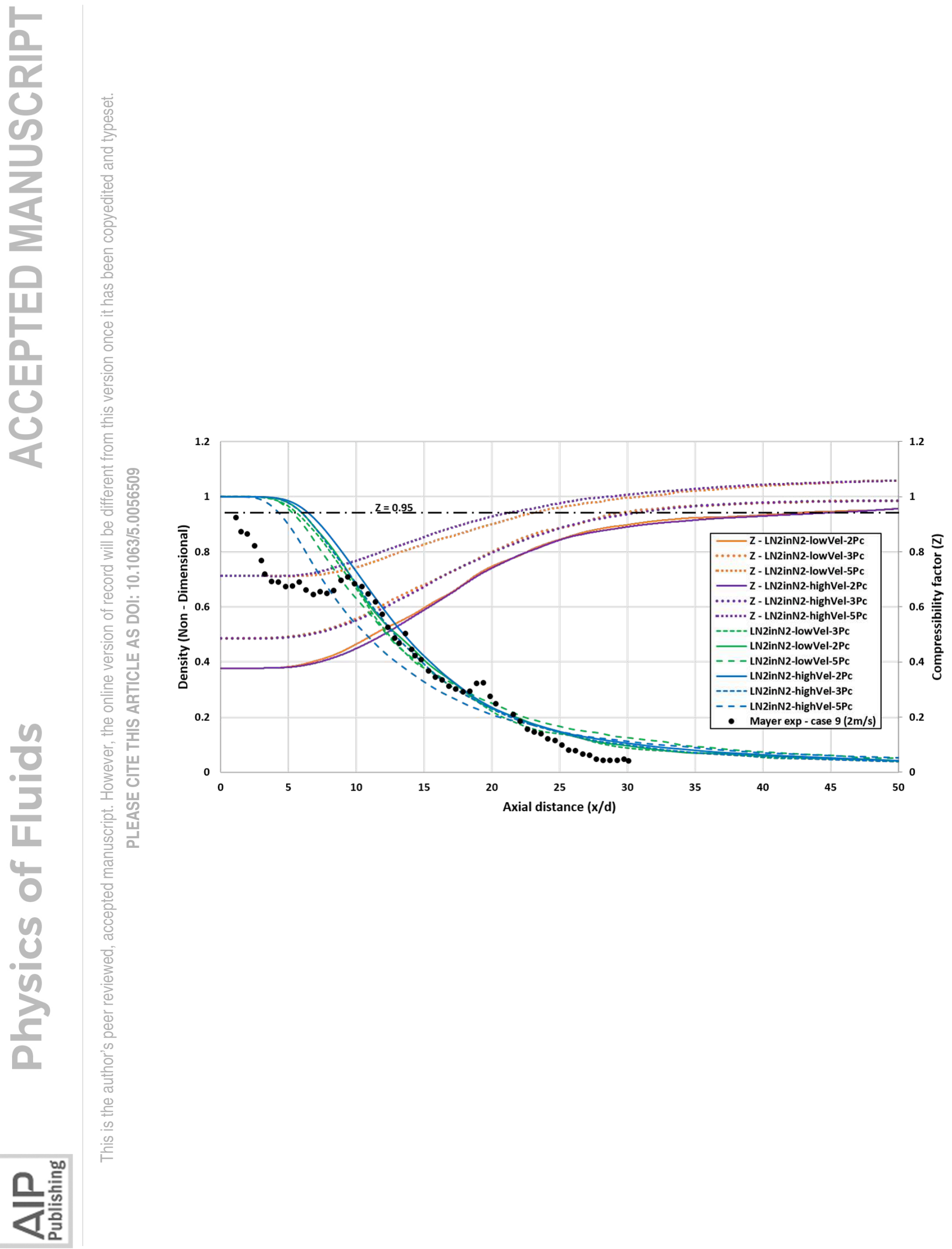

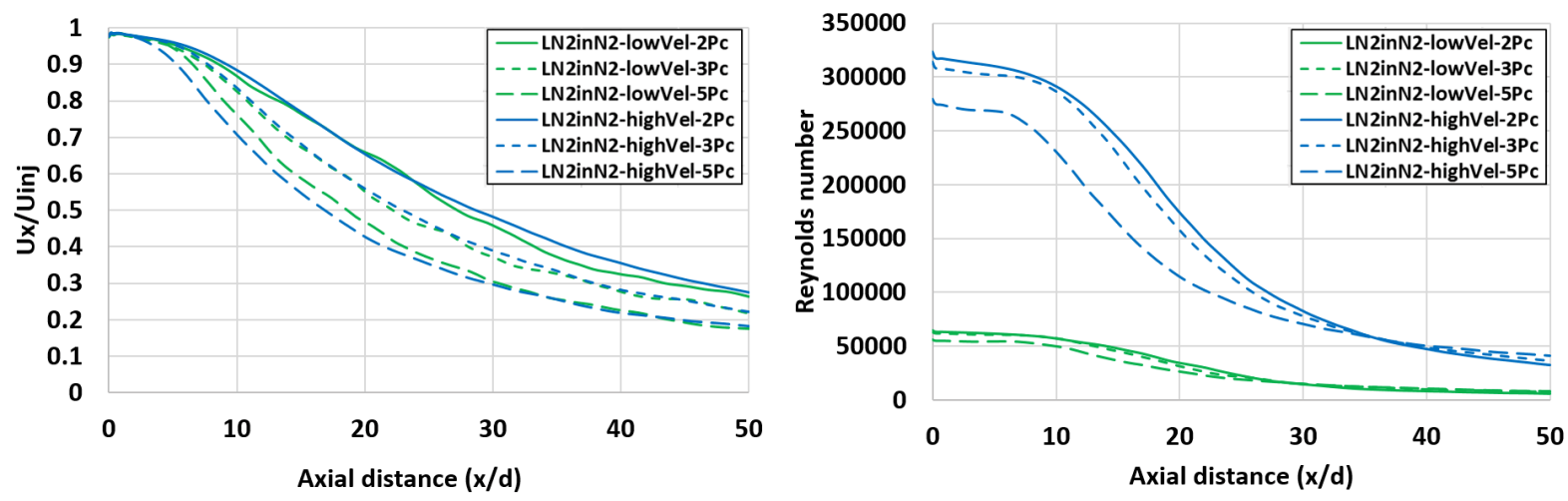

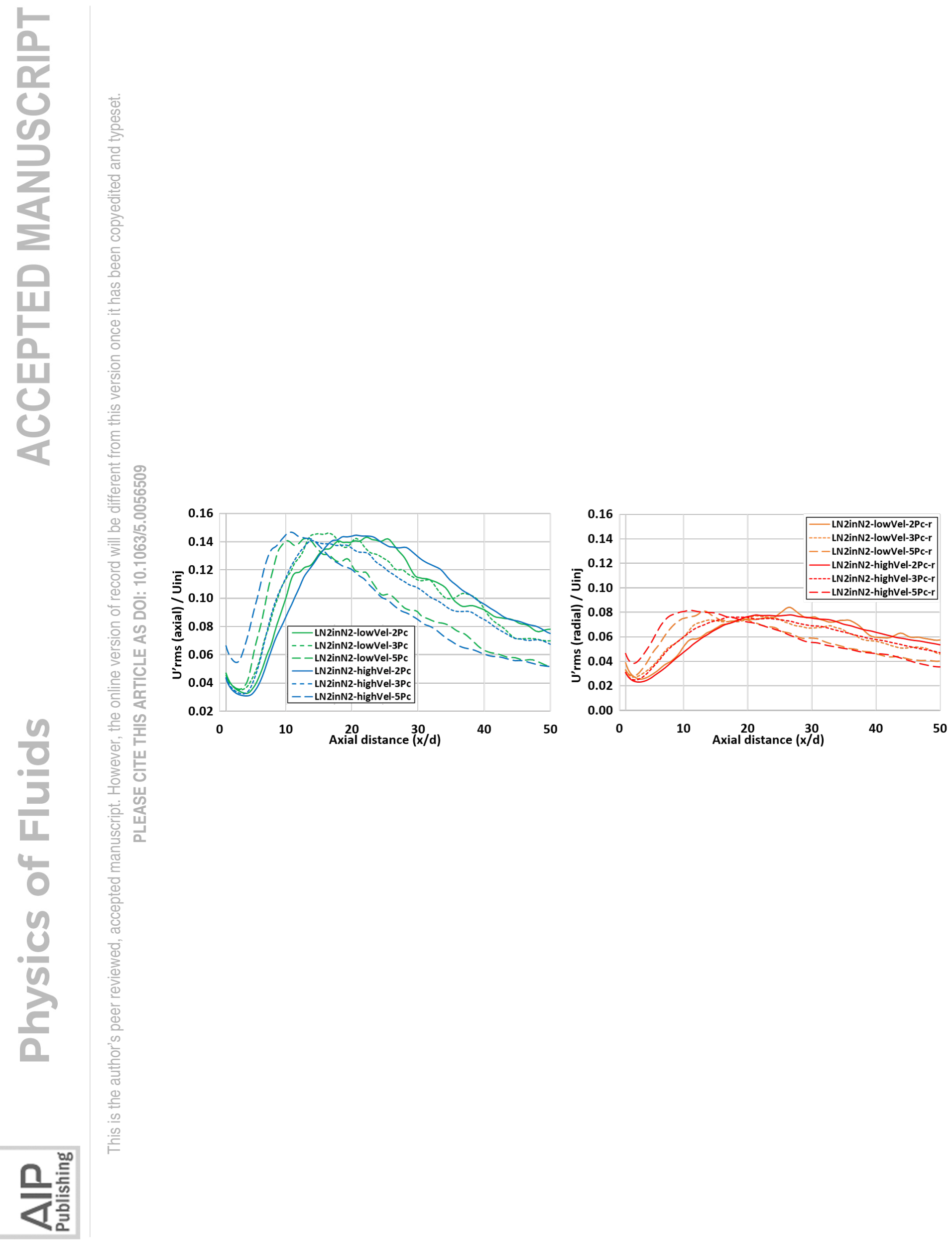


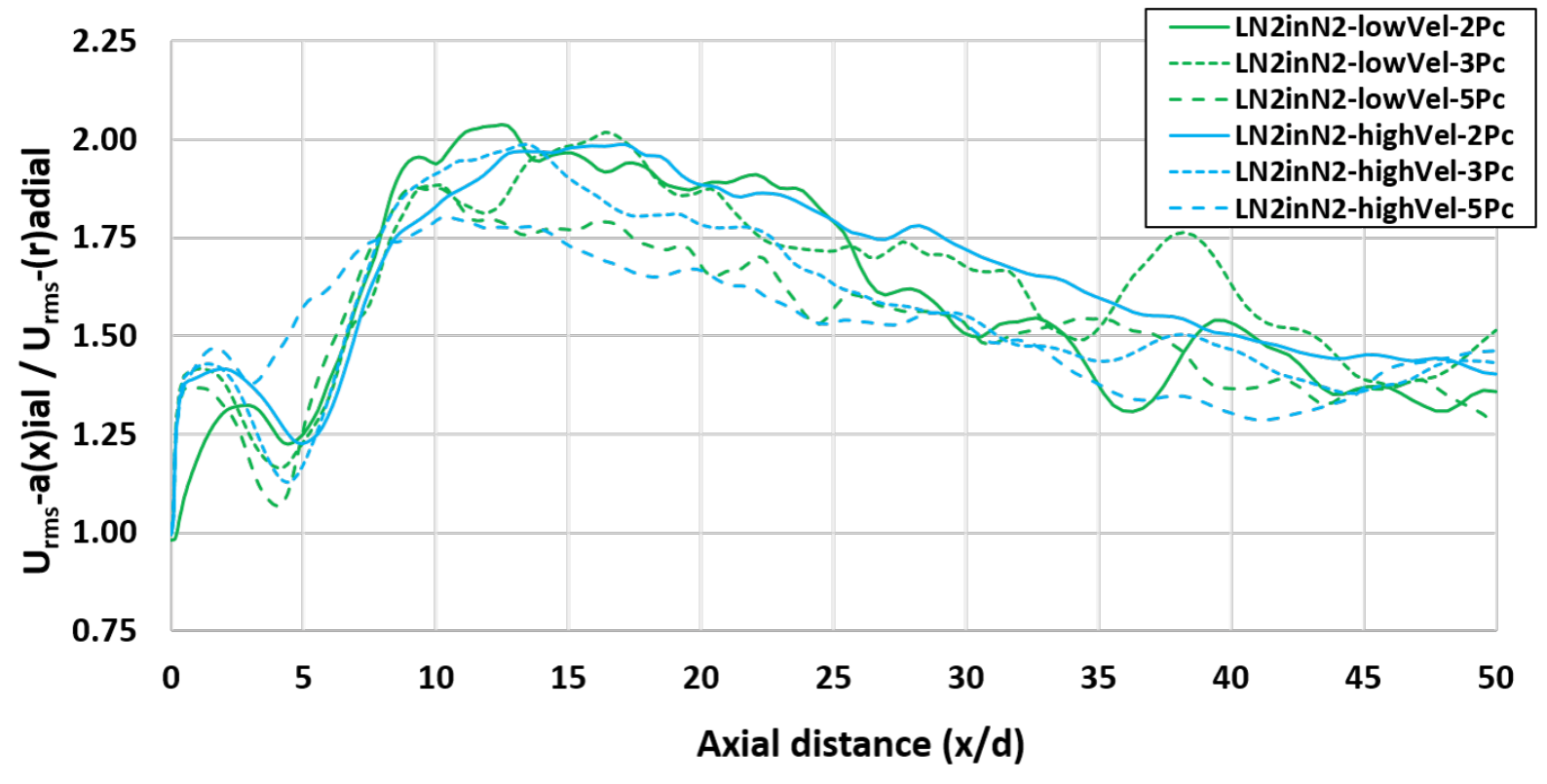



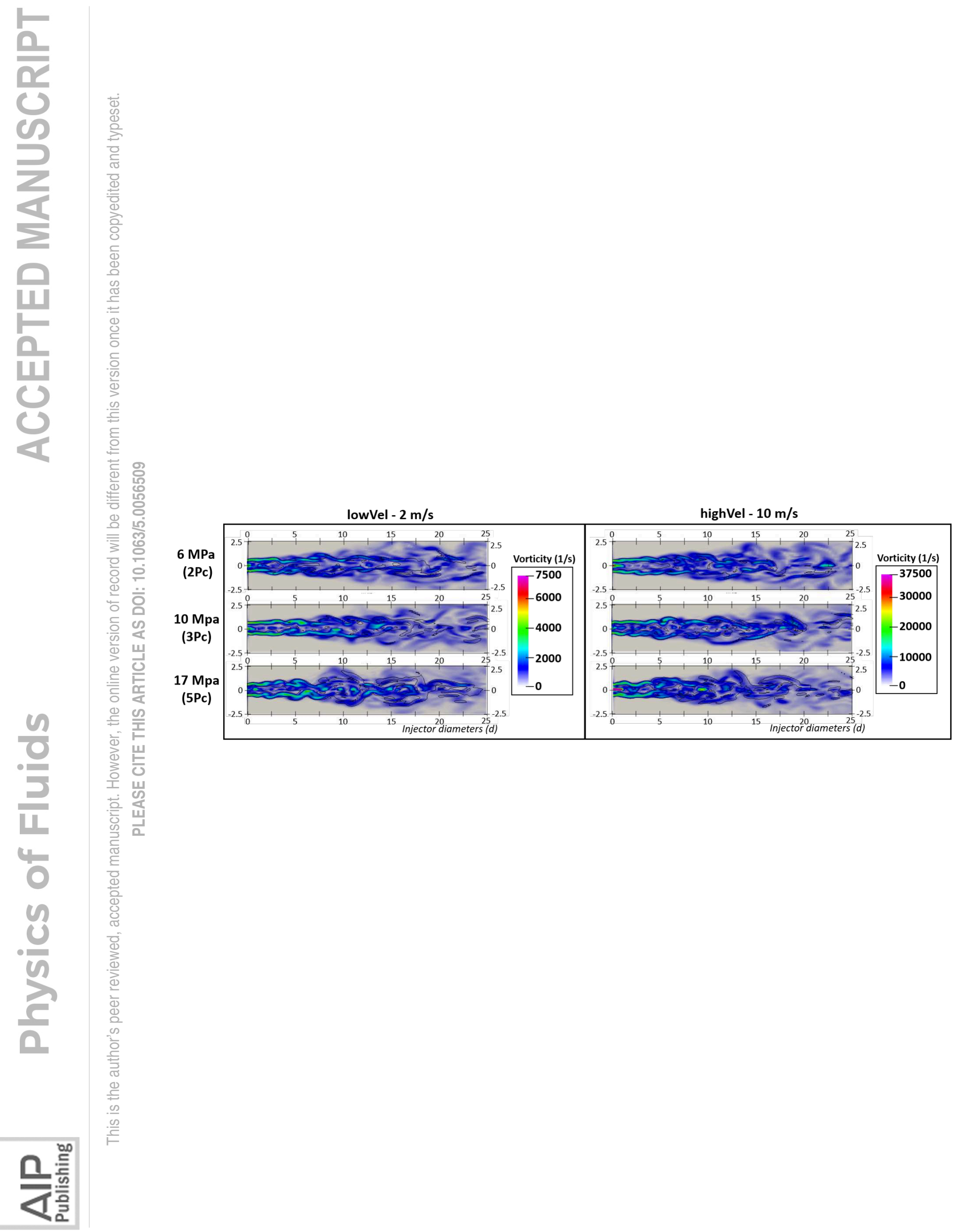

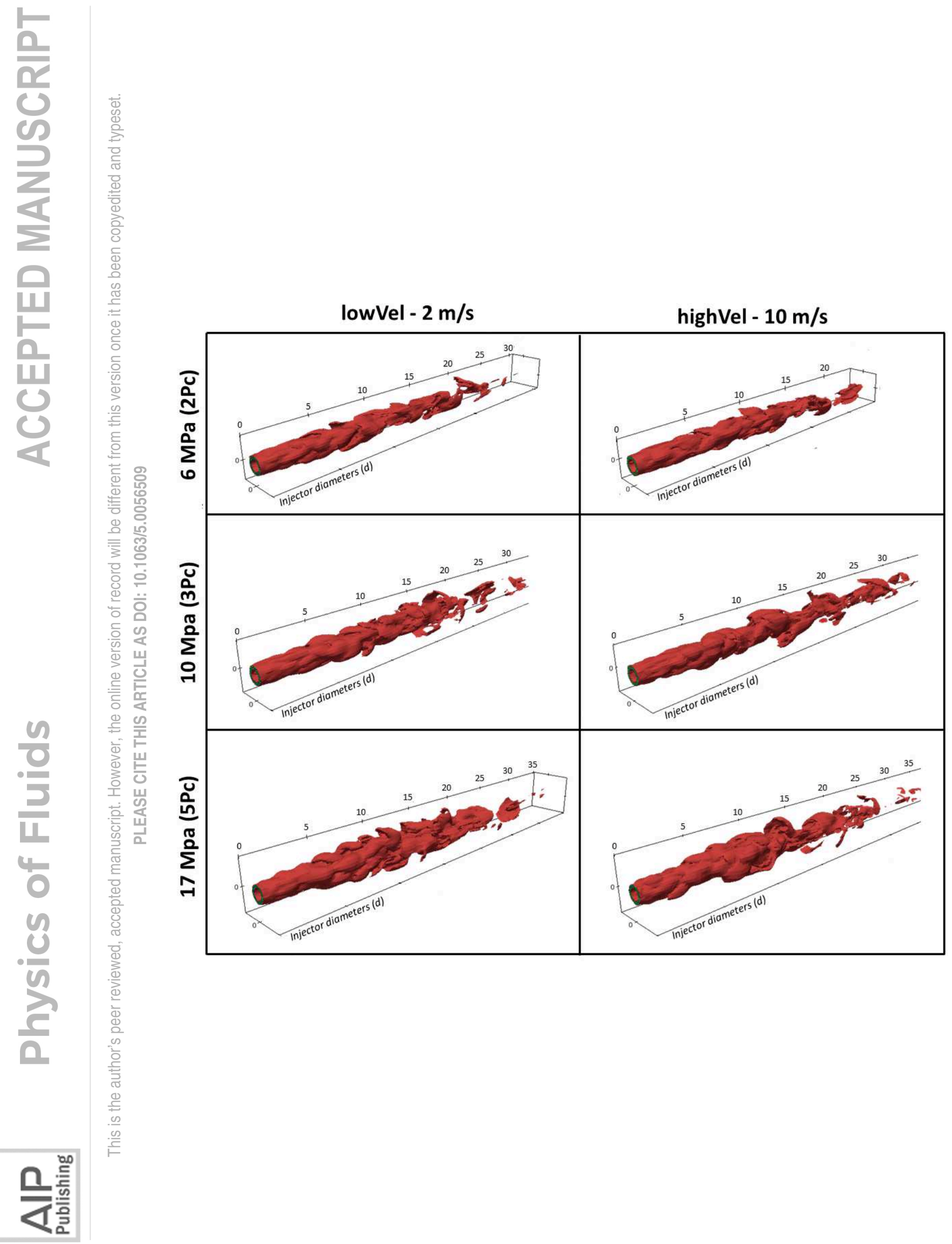


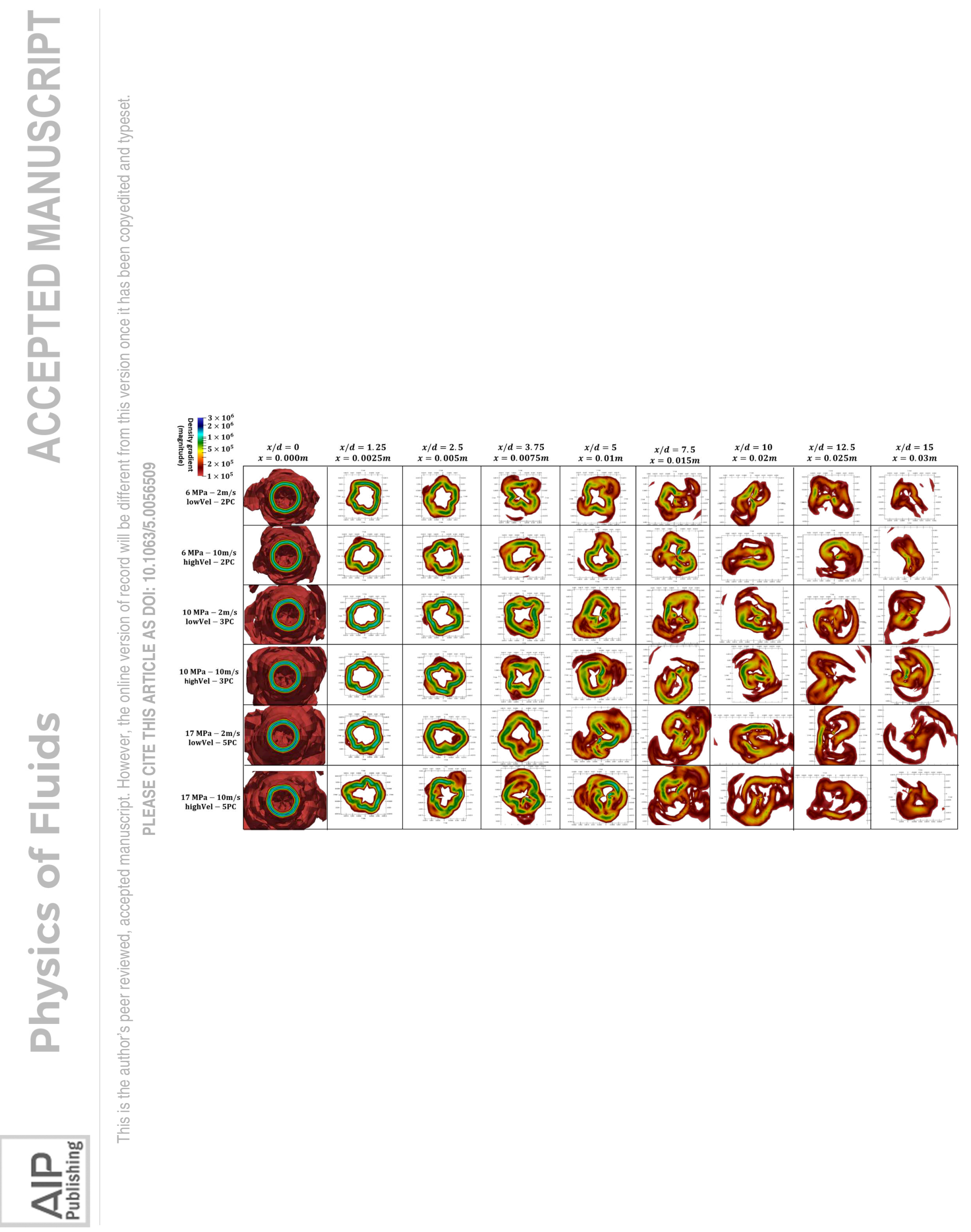




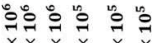

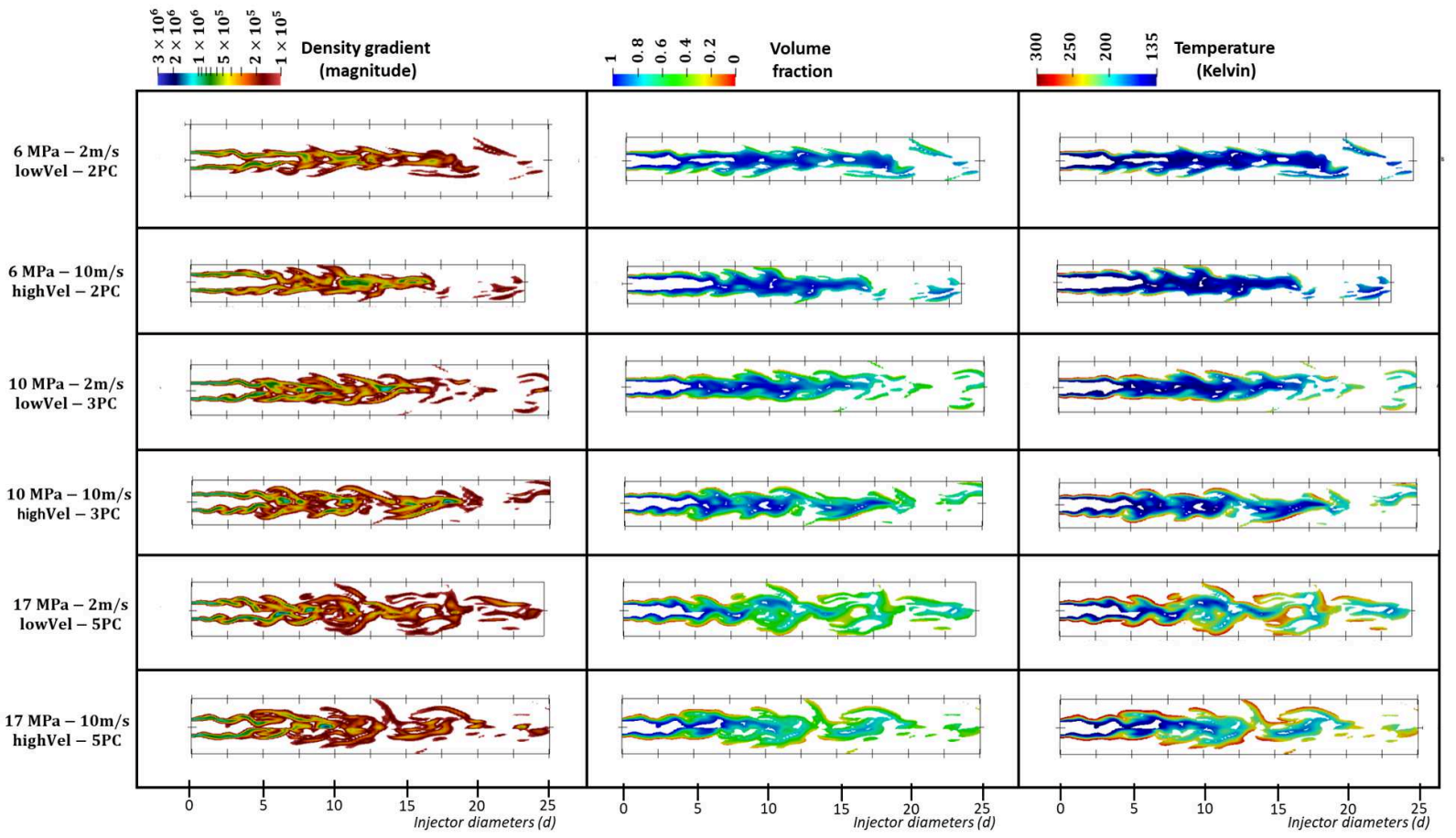




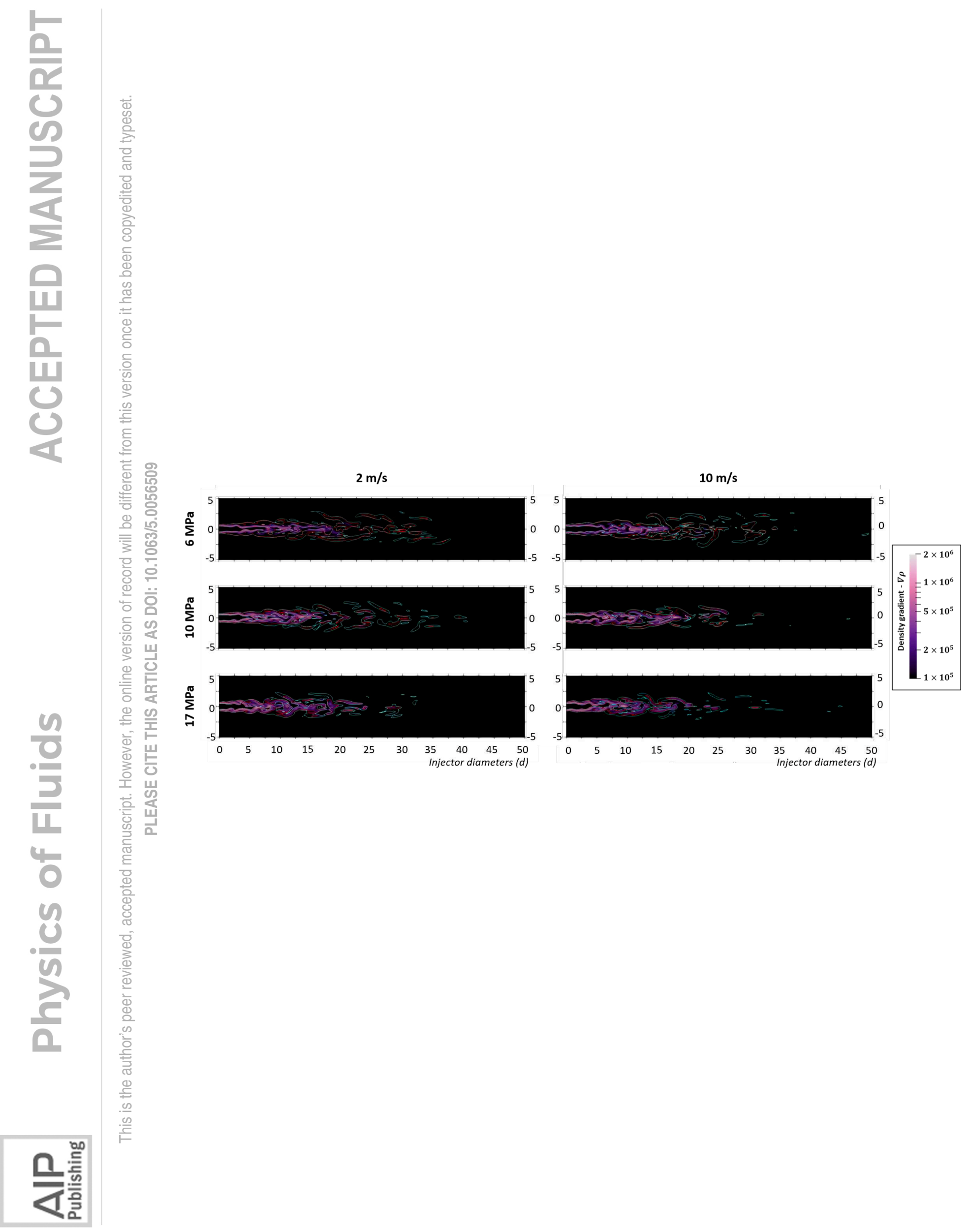



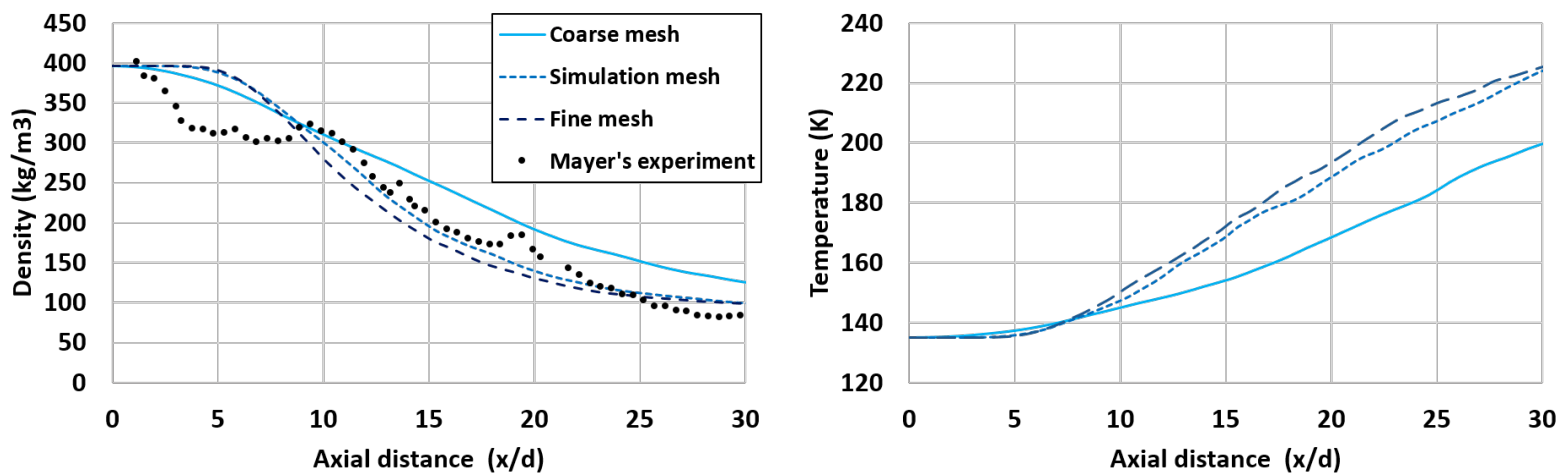